\title{
Maintenance-energy requirements and robustness of Saccharomyces cerevisiae at aerobic near-zero specific growth rates
}

\author{
Tim Vos, Xavier D. V. Hakkaart, Erik A. F. de Hulster, Antonius J. A. van Maris, Jack T. Pronk \\ and Pascale Daran-Lapujade*
}

\begin{abstract}
Background: Saccharomyces cerevisiae is an established microbial platform for production of native and non-native compounds. When product pathways compete with growth for precursors and energy, uncoupling of growth and product formation could increase product yields and decrease formation of biomass as a by-product. Studying nongrowing, metabolically active yeast cultures is a first step towards developing S. cerevisiae as a robust, non-growing cell factory. Microbial physiology at near-zero growth rates can be studied in retentostats, which are continuouscultivation systems with full biomass retention. Hitherto, retentostat studies on S. cerevisiae have focused on anaerobic conditions, which bear limited relevance for aerobic industrial processes. The present study uses aerobic, glucose-limited retentostats to explore the physiology of non-dividing, respiring S. cerevisiae cultures, with a focus on industrially relevant features.
\end{abstract}

Results: Retentostat feeding regimes for smooth transition from exponential growth in glucose-limited chemostat cultures to near-zero growth rates were obtained by model-aided experimental design. During 20 days of retentostats cultivation, the specific growth rate gradually decreased from $0.025 \mathrm{~h}^{-1}$ to below $0.001 \mathrm{~h}^{-1}$, while culture viability remained above $80 \%$. The maintenance requirement for ATP $\left(m_{\text {ATP }}\right)$ was estimated at $0.63 \pm 0.04 \mathrm{mmol}$ ATP (g biomass) $)^{-1} \mathrm{~h}^{-1}$, which is ca. $35 \%$ lower than previously estimated for anaerobic retentostats. Concomitant with decreasing growth rate in aerobic retentostats, transcriptional down-regulation of genes involved in biosynthesis and up-regulation of stress-responsive genes resembled transcriptional regulation patterns observed for anaerobic retentostats. The heat-shock tolerance in aerobic retentostats far exceeded previously reported levels in stationary-phase batch cultures. While in situ metabolic fluxes in retentostats were intentionally low due to extreme caloric restriction, off-line measurements revealed that cultures retained a high metabolic capacity.

Conclusions: This study provides the most accurate estimation yet of the maintenance-energy coefficient in aerobic cultures of $\mathrm{S}$. cerevisiae, which is a key parameter for modelling of industrial aerobic, glucose-limited fedbatch processes. The observed extreme heat-shock tolerance and high metabolic capacity at near-zero growth rates demonstrate the intrinsic potential of $S$. cerevisiae as a robust, non-dividing microbial cell factory for energy-intensive products.

Keywords: Yeast, Retentostat, Zero growth, Robustness, Heat-shock, Aerobic, Energetics, Maintenance

\section{Background}

The yeast Saccharomyces cerevisiae is an established microbial host for the production of native yeast

*Correspondence: p.a.s.daran-lapujade@tudelft.nl

Department of Biotechnology, Delft University of Technology, Van der Maasweg 9, $2629 \mathrm{HZ}$ Delft, The Netherlands metabolites as well as non-native products [1]. Production of many of these compounds, including phenylpropanoids, isoprenoids, heterologous proteins and lipids [2-4] from glucose requires a net input of ATP. The maximum ATP yield from glucose is obtained when its dissimilation occurs exclusively via respiration. In $S$. 
cerevisiae, a completely respiratory sugar metabolism requires aerobic conditions and sugar-limited cultivation at low to intermediate specific growth rates [5]. In industry, these requirements are usually met by sugar-limited, aerobic fed-batch cultivation. Due to oxygen-transfer and cooling constraints, aerobic fed-batch processes typically involve low specific growth rates [6, 7]. However, biomass-specific production rates $\left(\mathrm{q}_{\mathrm{p}}\right)$ of products whose biosynthesis from sugar requires a net input of ATP typically show a positive correlation with specific growth rate $[3,4,8,9]$. Understanding and, ultimately, breaking this correlation between growth and product formation by improving specific rates of product formation at low specific growth rates, is an important target for optimizing productivity and product yields in aerobic, sugar-limited fed-batch cultures.

In addition to the relation between $\mathrm{q}_{\mathrm{p}}$ and specific growth rate, microbial product formation at low specific growth rates is strongly influenced is by the metabolicenergy requirement of microorganisms for maintaining cellular integrity and viability. In a first analysis, this maintenance-energy requirement is often assumed to be growth-rate independent $[3,10]$. Distribution of carbon- and energy substrate over growth and cellular maintenance can then be described by the Pirt equation [11], which can be modified to include ATP-requiring product formation (see equation in Fig. 1). The Pirt equation describes how the fraction of the energy substrate that needs to be dissimilated to fulfil maintenance energy requirements increases as the specific growth rate in, for example, an aerobic, sugar-limited fed-batch process decreases. In slow-growing aerobic industrial fed-batch processes this increasing impact of maintenance requirements has a major negative impact on product yields and productivities [3].

Analysis of the physiology of extremely slow growing yeast cultures can provide relevant, quantitative information on the maintenance-energy requirements of $S$. cerevisiae and for developing this yeast into a non-growing cell factory [12-16]. Retentostats are continuous cultivation devices with full biomass retention that have been designed to study microbial physiology at near-zero growth rates $[17,18]$. Retentostat cultivation typically starts with a steady-state chemostat culture, operated at a low dilution rate. After reaching steady state, the chemostat culture is switched to retentostat mode by redirecting the effluent through a filter unit that ensures full biomass retention (Fig. 1). The constant, growth-limiting feed of glucose will then result in biomass accumulation $\left(C_{\mathrm{X}}\right)$, while the amount of substrate available per cell per unit of time decreases over time (Fig. 1). This decreased substrate availability results in decreasing specific substrate consumption rates $\left(\mathrm{q}_{\mathrm{S}}\right)$ which, after prolonged retentostat cultivation, asymptotically approach the cellular energy-substrate requirement for maintenance $\left(\mathrm{m}_{\mathrm{S}}\right)$. Since, in this situation, no energy-substrate is available

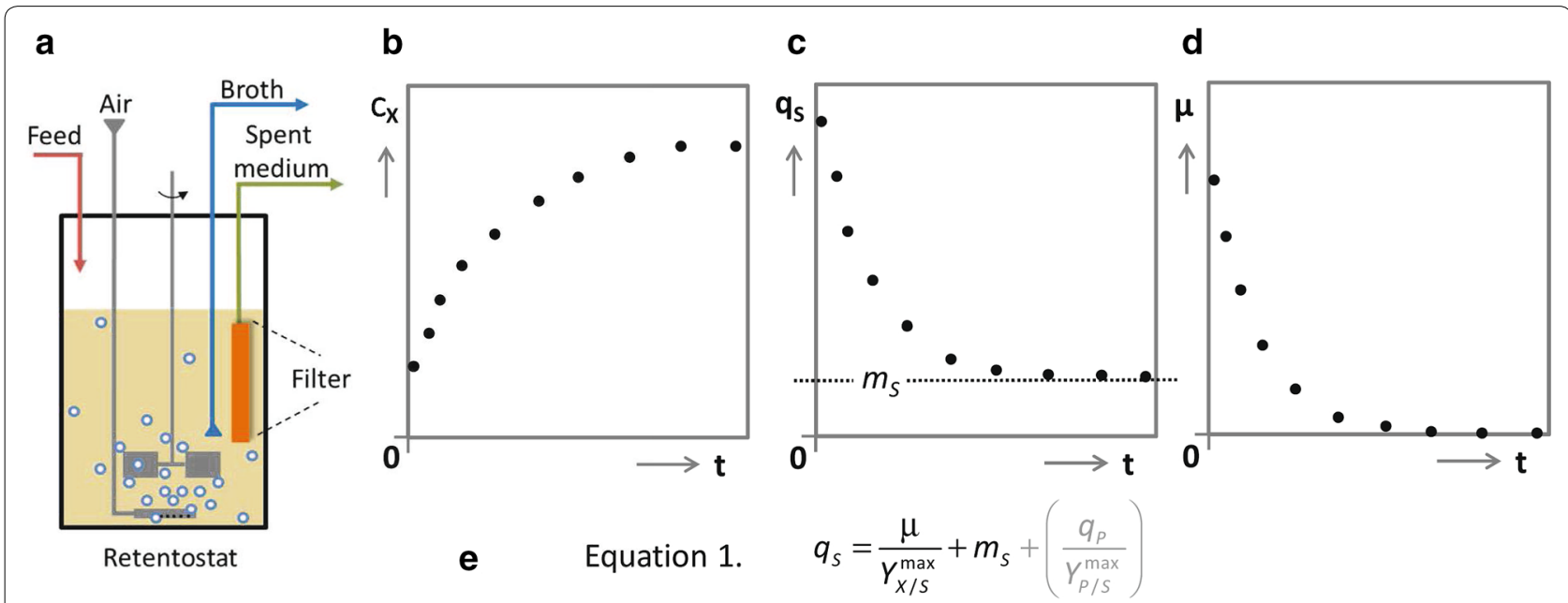

Fig. 1 Schematic representation of retentostat set-up and simulated profiles of biomass accumulation $\left(C_{X}\right)$, glucose consumption rate $\left(q_{S}\right)$ and specific growth rate $(\mu)$ during prolonged retentostat cultivation. The retentostat is a continuous bioreactor system in which the outflow can be switched from whole-broth removal to complete cell retention through a filter probe (a). After switching from chemostat cultivation to retentostat mode, biomass accumulates in the bioreactor $(\mathbf{b})$, which gradually decreases the glucose availability per unit of biomass. This decrease ultimately results (c) in specific glucose consumption rates that can only meet energy demands for cellular maintenance $\left(m_{s}\right)$, thereby causing near-zero specific growth rates $(\mathbf{d})$. The distribution of the carbon and energy source over growth, maintenance and product formation (not indicated in the plots) is mathematically captured by an extended Pirt equation (e), in which $Y_{x / 5}^{\max }$ is the maximum theoretical biomass yield, $\mathrm{q}_{\mathrm{p}}$ is the specific production rate of a product whose synthesis requires metabolic energy, and $Y_{\mathrm{P} / \mathrm{S}}^{\max }$ is the maximum theoretical yield of this product on substrate 
for growth, the specific growth rate $(\mu)$ asymptotically approaches zero (Fig. 1).

Retentostat cultures have mostly been used in the early 1990's to investigate the response of prokaryotes to extreme energy limitation. At extremely low growth rates, many bacteria, including Escherichia coli, display an alarmone-mediated stringent response. This coordinated response enables cultures to more efficiently withstand nutrient scarcity by down regulation of energy-intensive cellular processes and, therefore, a reduction of the maintenance-energy requirement [19-21]. Retentostats have recently been used to study the physiology of $S$. cerevisiae at near-zero growth rates under anaerobic conditions [12-16]. Even at extremely low specific growth rates, the maintenance requirement of $S$. cerevisiae in these anaerobic chemostat cultures was shown to be growthrate independent [12]. A decrease of the ATP-turnover of non-growing cultures was only observed when anaerobic, retentostat-grown $S$. cerevisiae cultures were switched to glucose starvation and energy metabolism became dependent on metabolism of storage carbohydrates [13]. Transcriptome responses during anaerobic retentostats encompassed many genes whose transcription was previously shown to be growth-rate correlated in faster growing cultures, as well as an increased expression of genes involved in resistance to a variety of stresses [14]. Consistent with the latter observation, yeast cells grown at low specific growth rates acquire a strongly increased robustness towards heat shock and an increased chronological life span [13, 22].

Since previous retentostat studies on S. cerevisiae were exclusively performed under anaerobic conditions, it remains unclear how oxygen availability affects its physiology at extremely low specific growth rates. Oxygen is known to have multiple effects on cellular biology. Even in S. cerevisiae, which has a rather low efficiency of oxidative phosphorylation, fully respiratory dissimilation of glucose yields eightfold more ATP than alcoholic fermentation, which is the sole dissimilatory pathway under anaerobic conditions [23]. This higher ATP yield supports higher biomass yields and, if the maintenance-requirement for ATP $\left(\mathrm{m}_{\text {АTP }}\right)$ is the same in aerobic and anaerobic cultures, should lead to a lower $\mathrm{m}_{\mathrm{S}}$ than observed in anaerobic cultures. Since biomass yield and maintenanceenergy requirement affect the dynamics of retentostats, these differences should also be taken into account in the design of feed regimes that result in a smooth transition from exponential growth to near-zero growth rates. Despite the industrial relevance of maintenance-energy requirements, accurate experimental estimates of $m_{S}$ and $\mathrm{m}_{\text {ATP }}$ for aerobic, sugar-limited cultures of S. cerevisiae on synthetic medium are not available. The assumption that $\mathrm{m}_{\mathrm{ATP}}$ in aerobic cultures is the same as in anaerobic cultures [24], can result in over- or underestimation of the $\mathrm{m}_{\mathrm{s}}$ of aerobic cultures. In anaerobic cultures, presence of the anaerobic growth factor oleic acid [25] and of ethanol and organic acids might increase $\mathrm{m}_{\text {ATP }}$. Similarly, detoxification of reactive oxygen species (ROS) and repair of ROS-induced damage may lead to increased maintenance energy requirements in aerobic cultures [26]. ROS, which can contribute to cellular aging, could also accelerate cell death of aerobic, non-dividing and chronologically aging yeast cultures [27]. A further question that remains to be addressed is whether and to what extent extremely slow-growing S. cerevisiae cultures retain a high metabolic capacity, which is a prerequisite for efficient product formation. Previous studies showed that glucose-limited aerobic cultures of $S$. cerevisiae retain a high capacity of glycolysis (the highway for sugar assimilation) at specific growth rates down to $0.05 \mathrm{~h}^{-1}$ [28], but no data are available on the glycolytic capacity at near-zero growth rates.

The goal of the present study is to quantitatively analyse maintenance-energy requirement, robustness and glycolytic capacity of $S$. cerevisiae in aerobic cultures grown at near-zero growth rate. To this end, regimes for aerobic retentostat cultivation were designed and implemented that enabled a smooth transition from exponential growth to near-zero growth rates. In addition to quantitative physiological analyses, transcriptome analysis was performed to investigate cellular responses to near-zero growth in aerobic cultures and to compare these with previously published transcriptome data obtained from anaerobic retentostats.

\section{Results}

Design of a regime for smooth transition to near-zero growth rates in aerobic retentostats

Growth rate dynamics and biomass accumulation in retentostat cultures mainly depend on two conditiondependent and strain-specific parameters: the theoretical maximal biomass yield $\left(\mathrm{Y}_{\mathrm{X} / \mathrm{S}}^{\max }\right)$ and the maintenance coefficient $\left(\mathrm{m}_{\mathrm{S}}\right)$. To predict the impact of these parameters on growth dynamics in aerobic retentostat cultures, a model based on the Pirt definition of resource allocation (see Fig. 1) was used. $Y_{X / S}^{\max }$ was estimated from published data on aerobic, glucose-limited chemostat cultures of the $S$. cerevisiae strain used in this study $\left(0.5 \mathrm{~g} \mathrm{~g}^{-1}\right.$ [28]). Since no accurate estimates for the aerobic $\mathrm{m}_{\mathrm{S}}$ are available, model-based simulations were performed with a range of $m_{S}$ values that were based on the $m_{S}$ calculated from anaerobic retentostat experiments (biomass-specific glucose consumption for maintenance: $0.5 \mathrm{mmol} \mathrm{g}_{\mathrm{X}}^{-1} \mathrm{~h}^{-1}$, [12]) and assuming a $\mathrm{P} / \mathrm{O}$ ratio of 1.0 for aerobic, respiring cultures of $S$. cerevisiae $[23,29]$, which leads to an eightfold higher ATP yield from respiratory sugar dissimilation than from alcoholic fermentation. 
Initial model simulations were performed based on the assumption that no loss of viability occurs during retentostat cultivation and with the same feed regime that was previously used for anaerobic retentostats (constant dilution rate of $0.025 \mathrm{~h}^{-1}$ and a glucose concentration in the feed of $20 \mathrm{~g} \mathrm{~L}^{-1}$ [12]). This resulted in a predicted accumulation of biomass to a concentration of ca. $45 \mathrm{~g} \mathrm{~L}^{-1}$, Fig. 2, blue line), which was considered to present a substantial risk of clogging the filter unit. Moreover, in this simulation, near-zero growth rates (i.e. specific growth rates below $0.001 \mathrm{~h}^{-1}$ ) were only reached after multiple weeks of operation (Fig. 2, blue line), which was considered to be impracticable.

Near-zero growth rates can be reached faster by decreasing the glucose concentration in the medium for the retentostat culture $\left(C_{S, M R}\right)$ compared to the glucose concentration in the medium for the chemostat culture $\left(\mathrm{C}_{\mathrm{S}, \mathrm{MC}}\right)$. However, care should be taken to avoid scenarios in which the glucose supply changes suddenly or transiently decreases below the culture's maintenance-energy demand, which might affect cellular viability. Introduction of an additional medium mixing vessel (Fig. 2), allowed for a controlled, smooth transition of the ingoing glucose concentration $\left(\mathrm{C}_{\mathrm{S}, \mathrm{in}}\right)$ into the retentostat culture, whilst maintaining a constant flow of medium $\left(\phi_{\mathrm{V}}\right)$ and thereby a constant dilution rate. To incorporate the mixing vessel the model was expanded with Eq. 2 and simulations were performed for experimental design of $\mathrm{C}_{\mathrm{S}, \mathrm{MR}}$ and the volume of the mixing vessel $\left(\mathrm{V}_{\mathrm{S}}\right.$ in liters) (Fig. 2).

$$
\frac{d C_{S, \text { in }}}{d t}=\frac{\phi_{V}}{V_{S}} C_{S, M R}-\frac{\phi_{V}}{V_{S}} C_{S, i n}
$$

Figure 2 depicts the modelling output when $C_{S, M R}$ equals $\mathrm{C}_{\mathrm{S}, \mathrm{MC}}$ (blue lines) and when $\mathrm{C}_{\mathrm{S}, \mathrm{MR}}$ was decreased to 7.5 or $5 \mathrm{~g} \mathrm{~L}^{-1}$ (solid red and green lines, respectively) assuming an $\mathrm{m}_{\mathrm{S}}$ of $0.011 \mathrm{~g} \mathrm{~g}_{\mathrm{X}}^{-1} \mathrm{~h}^{-1}$.

In the simulations, values of $\mathrm{C}_{\mathrm{S}, \mathrm{MR}}$ of $5 \mathrm{~g} \mathrm{~L}^{-1}$ and lower resulted in 'negative growth', indicating that the model predicted glucose starvation and cell death. Since, in extremely slow growing cultures, glucose is predominantly used for maintenance, growth dynamics in retentostats are particularly sensitive to variations in $\mathrm{m}_{\mathrm{S}}$. Accordingly, a $20 \%$ change in $\mathrm{m}_{\mathrm{S}}$ resulted in a fivefold difference in the predicted specific growth rates after 20 days of retentostat cultivation (dashed lines in Fig. 2). Based on these simulations, operational conditions were chosen such that the prediction complied to the following requirements: (i) near-zero growth rates $\left(\mu<0.001 \mathrm{~h}^{-1}\right)$ achieved within 2 weeks of retentostat cultivation, (ii) prevention of sudden changes in $\mathrm{q}_{\mathrm{s}}$ and glucose starvation, (iii) the conditions led to a sizeable difference between initial and final biomass concentrations, (iv) previous criteria met for a range of $\mathrm{m}_{\mathrm{S}}$ values, and (v) final biomass concentration kept below $30 \mathrm{~g} \mathrm{~L}^{-1}$ to prevent filter clogging (Fig. 2, red line). The chosen operational conditions are described in Fig. 2, and correspond to the red line.

\section{Maintenance-energy requirements in aerobic retentostat cultures}

In four independent retentostat cultures, biomass accumulated reproducibly over a period of ca. 20 days. The final biomass concentrations were ca. threefold higher than those in the preceding chemostat culture (Fig. 3a). However, the experimentally observed biomass accumulation was substantially higher than predicted from model simulations (Fig. 3a). One factor that might contribute to this apparent discrepancy was the biomass viability which, in the model simulations, was assumed to remain at $100 \%$ throughout the retentostats experiments. Indeed, flow-cytometric analysis of cellular integrity indicated that, over 20 days of retentostat cultivation, culture viability decreased to ca. 85 \% (Fig. 3b). Colony-forming unit counts confirmed that ca. $70 \%$ of the cells in the population were able to sustain growth after 20 days in retentostat culture. This apparent loss of cells' capacity to divide contrasted with the retention of cellular integrity and has been previously reported for anaerobic retentostat cultures [15]. It may result from various factors, such as the irreversible degradation of macromolecules necessary for duplication, but may also result from loss of reproductive capacity during CFU plating assays. To prevent the risk of underestimating culture viability, viable biomass concentrations were therefore calculated based on flow cytometry-based viability assays (Fig. 3a). Based on these observations, a low but significant death rate of $4.7 \cdot 10^{-4} \mathrm{~h}^{-1}$ was calculated. However, correcting for viability only explained part of the difference between the observed and modelled biomass accumulation profiles.

As mentioned above, the exact value of $\mathrm{m}_{\mathrm{S}}$ is expected to have a strong impact on biomass accumulation profiles in retentostat cultures. Assuming specific growthrate independent maintenance, the aerobic $\mathrm{m}_{\mathrm{S}}$ was estimated from the calculated specific growth rate and glucose consumption rates of $S$. cerevisiae in the aerobic retentostats, using biomass concentrations corrected for viability (Fig. 4a). During 20 days of retentostat cultivation, specific growth rates in all four replicate experiments decreased from $0.025 \mathrm{~h}^{-1}$ in steady-state to values below $8 \cdot 10^{-4} \mathrm{~h}^{-1}$, representing doubling times of over 36 days. The average specific glucose-consumption rate converged to $0.039 \pm 0.003 \mathrm{mmol} \mathrm{g}_{\mathrm{X}}^{-1} \mathrm{~h}^{-1}$, representing the cellular substrate requirement exclusively necessary for maintenance energy purposes (Fig. 4b). Considering an in vivo P/O ratio in S. cerevisiae of 1.0 [23], the aerobic ATP requirement of $S$. cerevisiae for maintenance $\left(\mathrm{m}_{\text {АTP }}\right)$ 

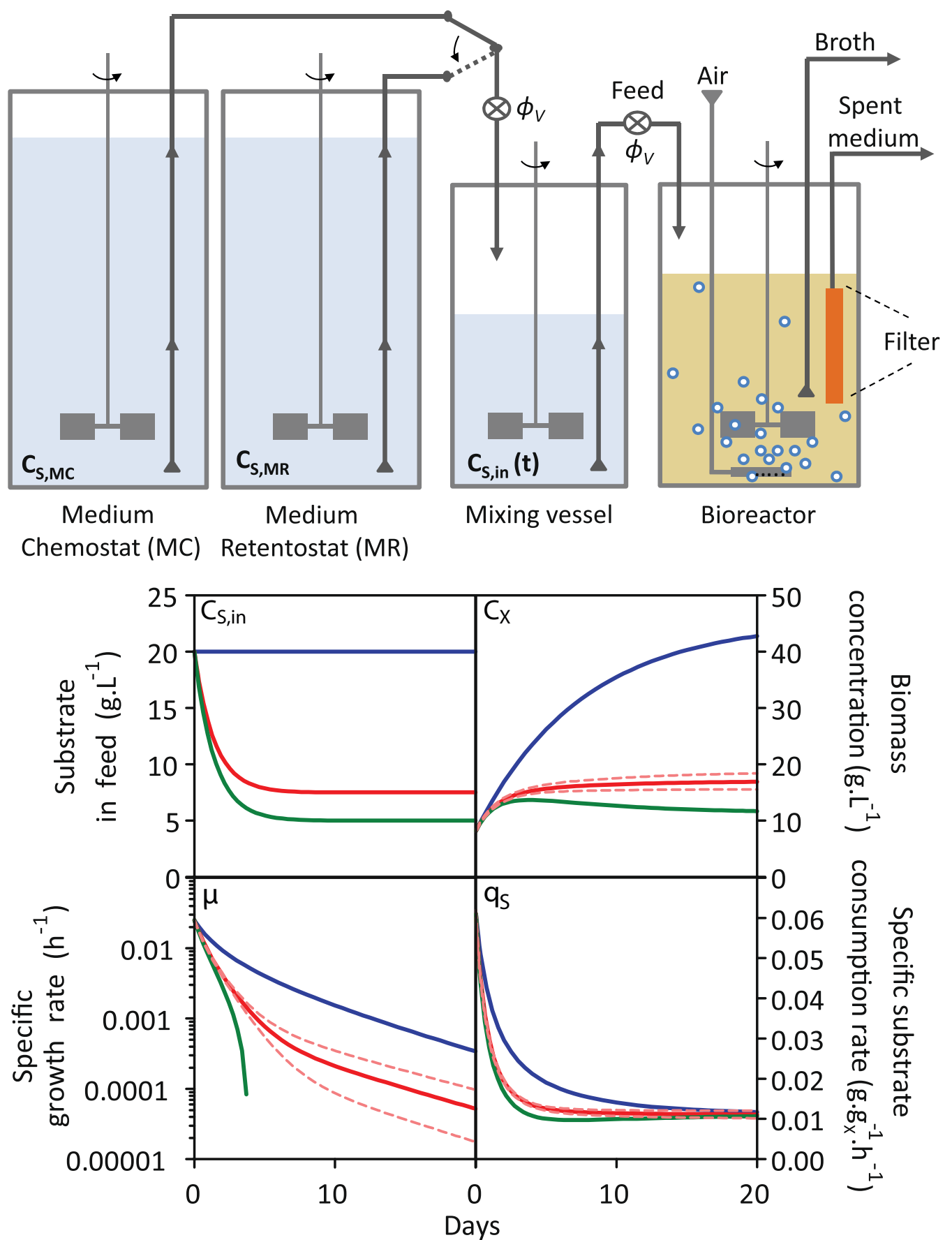

Fig. 2 Setting up aerobic retentostat cultures. a Retentostat cultures (bioreactors) were started from a steady-state chemostat culture with an ingoing glucose concentration $\left(C_{S, M C}\right)$ of $20 \mathrm{~g} \mathrm{~L}^{-1}$. At the start of the retentostats $(t=0 \mathrm{~h})$, the feed to the mixing vessel was switched to the medium reservoir for the retentostat cultivation (as indicated by the arrow). The process was modelled for three different concentrations of glucose in the medium reservoir for the retentostat cultures $\left(C_{S, M R}\right)$. b Profiles of biomass concentration $\left(C_{X}\right)$, specific glucose consumption rate $\left(q_{s}\right)$ and specific growth rate $(\mu)$ in time were predicted with a mathematical model, based on glucose concentration in the feed coming from the mixing vessel. Blue lines indicate a scenario in which $C_{S, M C}=C_{S, M R}=20 \mathrm{~g} \mathrm{~L}^{-1}$, green lines indicate $C_{S, M R}=5 \mathrm{~g} \mathrm{~L}^{-1}$, and red lines indicate $C_{S, M R}=7.5 \mathrm{~g} \mathrm{~L}^{-1}$. Dotted lines indicate simulations for which $10 \%$ higher or lower maintenance values were considered in the model (see "Methods" section). The operational conditions applied in the experiments in this study correspond to the red lines 

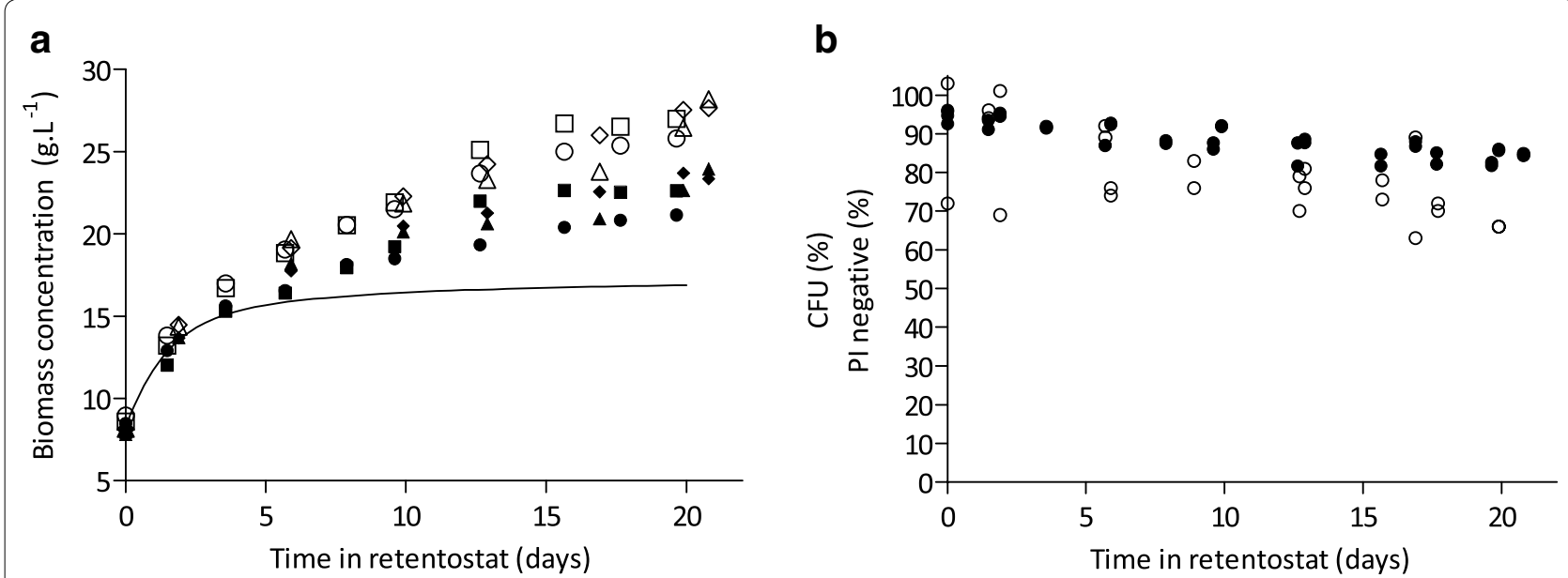

Fig. 3 Biomass accumulation and culture viability during prolonged retentostat cultivation. a Predicted biomass accumulation profile (line), measured biomass dry weight concentrations (open symbo/s), and viable biomass concentration (closed symbo/s) from four replicate retentostat cultures. b Culture viability estimated by flow cytometric analysis of propidium iodide-stained cells (closed symbo/s), and viability estimated from colonyforming unit counts (open symbols)
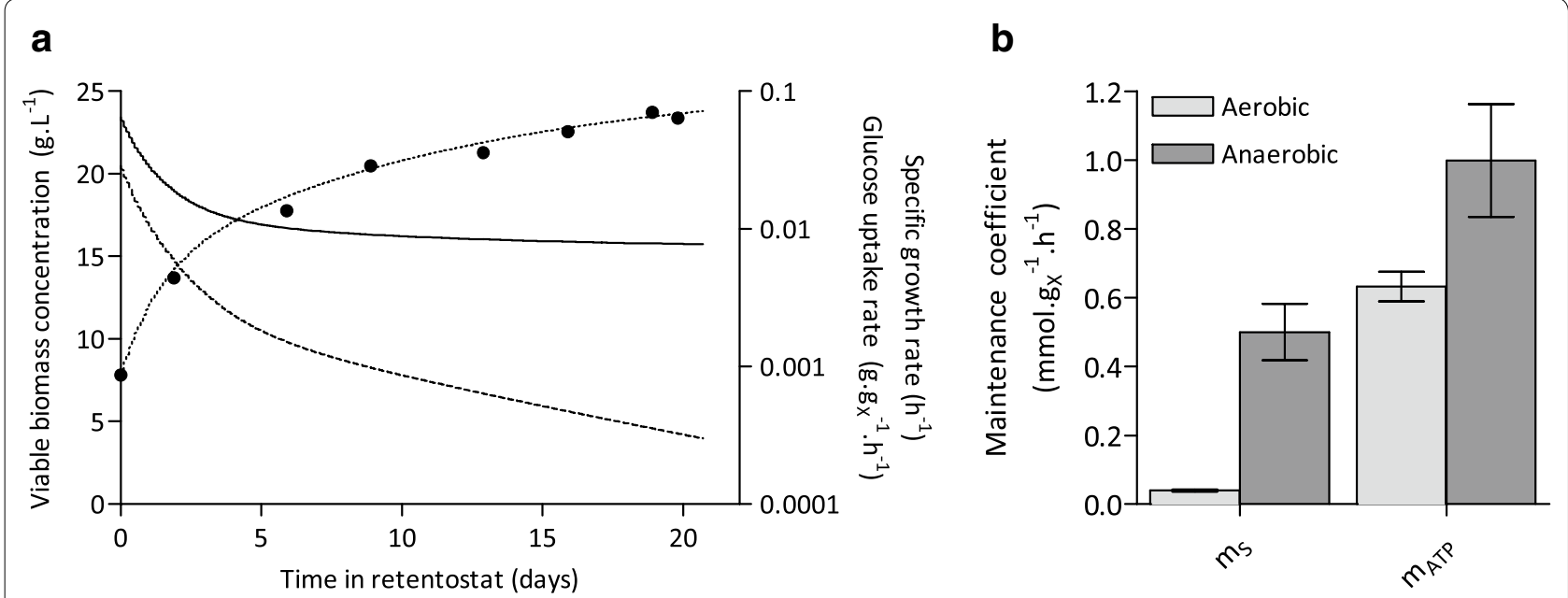

Fig. 4 Growth kinetics and $m_{S}$ in retentostat cultures. a Specific glucose-consumption rate ( $q_{s}$, solid line) and specific growth rate ( $\mu$, dashed line) calculated by non-linear regression of the accumulation of viable- and total biomass over time (see "Methods" section). The closed symbols and dotted line represent the viable biomass and linear regression of the viable biomass, respectively. Data are shown for a single representative retentostat experiment. b Glucose and ATP requirements for maintenance $\left(m_{S}\right.$ and $m_{\text {ATP, }}$ respectively) of aerobic and anaerobic retentostat cultures (anaerobic data obtained from [12]). The aerobic $m_{\text {ATP }}$ was calculated based on a P/O ratio of respiring S. cerevisiae cultures of 1.0 [23]

calculated from these experiments was $0.63 \pm 0.04 \mathrm{mmol}$ ATP $g_{\mathrm{X}}^{-1} h^{-1}$. This value is ca. $30 \%$ lower than the $\mathrm{m}_{\text {ATP }}$ previously estimated from anaerobic retentostats cultures [12] (Fig. 4b).

\section{Transcriptional reprogramming in aerobic retentostats: involvement of 'growth-rate responsive' genes}

Over the course of the aerobic retentostat experiments, 1375 genes (ca. one-fifth of the genome) were differentially expressed. In comparison, aerobic batch cultures transitioning from exponential growth, through a postdiauxic phase, into stationary phase, resulted in 1690 differentially expressed genes (using the same analysis software and statistical criteria as in the present work, Additional file 1) [34]. One third (458 genes) of the 1375 genes identified in the present retentostat dataset overlapped with the aerobic batch dataset. The 1375 differentially expressed genes identified in the present study could be separated in two clusters with clear, specificgrowth-rate dependent transcript profiles (Fig. 5). Cluster 

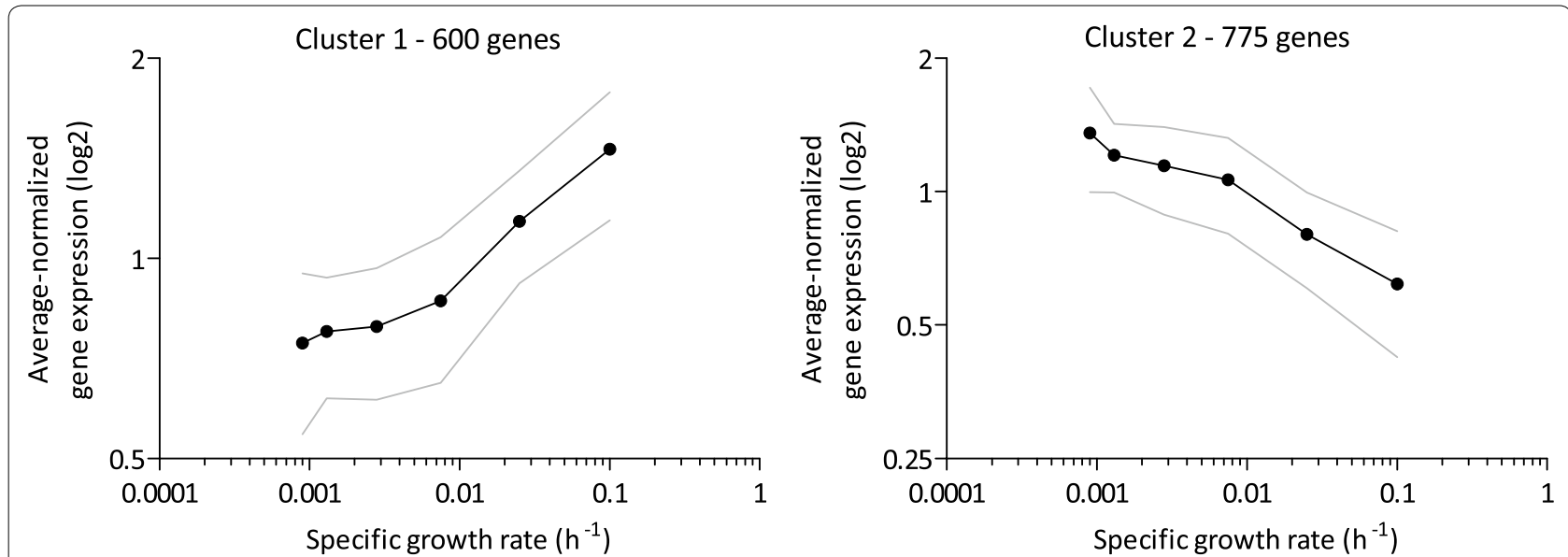

Fig. 5 K-mean clustering of the 1375 genes with significant growth-rate dependent expression profiles. Retentostat data were combined with data from aerobic glucose-limited chemostats grown at $\mu=0.10 \mathrm{~h}^{-1}$ (see "Methods" section). The p value threshold for significant differential expression was set to 0.01. For each cluster, averaged-normalized expression values are depicted as a function of specific growth rate (see "Methods" section). Grey dotted lines show the standard deviation of averaged expression values

1 harboured 600 genes whose expression displayed a positive correlation with specific growth rate (Fig. 5). As anticipated, this cluster showed an overrepresentation of genes involved in typical growth-related processes, including protein, ribosome, amino acid, nucleotide and lipid biosynthesis (Table 1). Consistent with this observation, cluster 1 also showed an overrepresentation of genes whose expression is controlled by transcription factors that are involved in this response: Fhl1, Rap1 and Sfp1, Gcn4 and Met32 (Table 1). Genes involved in sterol metabolism (including 15 of the 19 ERG genes involved in ergosterol synthesis) and pentose-phosphate pathway were also overrepresented in cluster 1 . Cluster 2 grouped the remaining 775 differentially expressed genes, whose transcript levels showed a negative correlation with specific growth rate (Fig. 5). This cluster was most strongly enriched for genes involved in stress response, and more specifically targets of Skn7 and Cad1, as well as for genes involved in signal transduction and protein turnover (Table 1). Accordingly, cluster 2 was strongly enriched for targets of the stress-responsive transcription factor pair Msn2/Msn4 (55 out of 166 genes, p value $4 \cdot 2910^{-11}$ ) [30].

A positive correlation with specific growth rate of the expression levels of genes involved in anabolic processes and a negative correlation of those of stress-responsive genes, was previously shown in aerobic chemostat cultures grown at specific growth rates of $0.05 \mathrm{~h}^{-1}$ and above [31-33]. Clusters 1 and 2 showed a substantial overlap with these previously identified sets of growthrate responsive genes (Additional file 2).

To investigate how cellular responses to near-zero growth rates differed between aerobic and anaerobic cultures, we compared transcriptome data from the present study with those obtained in a previous transcriptome analysis of anaerobic retentostats of the same S. cerevisiae strain [14]. Anaerobic retentostat cultivation yielded 2661 differentially expressed genes, based on the same range of specific growth rates and applying the same statistical criteria as in the present study. This number of genes is almost twofold higher than observed in the aerobic retentostats (Additional file 3). Synthetic medium, $\mathrm{pH}$ and temperature in the anaerobic retentostats were the same as those used in the present study, except for the addition of the anaerobic growth factors Tween-80 (a source of oleic acid) and ergosterol in the previous study.

Differences in the responses of anaerobic and aerobic retentostats were investigated by identifying genes that showed a specific transcriptional response to near-zero growth rates in either aerobic or anaerobic retentostats (Fig. 6). Among 182 genes whose expression increased at extremely low growth rates in anaerobic retentostat cultures, only functional categories related to aerobic respiration, were significantly enriched (Fig. 6). 31 out of 74 genes involved in the cellular function aerobic respiration were specifically up-regulated in anaerobic retentostats, including $8 \mathrm{COX}$ genes, which encode subunits of the mitochondrial inner-membrane cytochrome c oxidase. Genes involved in this functional category were not overrepresented among the responsive genes identified in aerobic retentostat cultures, indicating that up-regulation of respiration-related genes is a specific adaptation to anaerobic slow growing and/or aging cultures. Among the 686 genes whose expression showed a reduced expression at near-zero growth rates under anaerobic 
Table 1 Overrepresentation of functional categories among the two clusters of differentially expressed genes (see Fig. 5)

\begin{tabular}{|c|c|c|c|c|c|}
\hline & & Functional category & $K^{a}$ & $\mathrm{~N}^{\mathbf{b}}$ & p value $^{c}$ \\
\hline \multirow[t]{37}{*}{ Cluster 1} & \multirow[t]{17}{*}{ MIPS $^{d}$} & Protein synthesis & 138 & 511 & $4.98 \cdot 10^{-31}$ \\
\hline & & Ribosomal proteins & 95 & 277 & $4.29 \cdot 10^{-29}$ \\
\hline & & Ribosome biogenesis & 106 & 343 & $2.46 \cdot 10^{-28}$ \\
\hline & & Amino acid metabolism & 69 & 243 & $3.31 \cdot 10^{-15}$ \\
\hline & & Metabolism & 221 & 1530 & $5.87 \cdot 10^{-11}$ \\
\hline & & Metabolism of the aspartate family & 26 & 64 & $1.75 \cdot 10^{-8}$ \\
\hline & & Metabolism of methionine & 18 & 36 & $3.52 \cdot 10^{-7}$ \\
\hline & & Tetracyclic and pentacyclic triterpenes metabolism & 16 & 36 & $2.84 \cdot 10^{-5}$ \\
\hline & & Purine nucleotide/nucleoside/nucleobase metabolism & 22 & 66 & $4.58 \cdot 10^{-5}$ \\
\hline & & Nucleotide/nucleoside/nucleobase metabolism & 48 & 230 & $4.81 \cdot 10^{-5}$ \\
\hline & & Isoprenoid metabolism & 16 & 41 & $2.55 \cdot 10^{-4}$ \\
\hline & & Sulfur metabolism & 7 & 8 & $3.51 \cdot 10^{-4}$ \\
\hline & & Sulfate assimilation & 7 & 8 & $3.51 \cdot 10^{-4}$ \\
\hline & & Metabolism of the cysteine-aromatic group & 23 & 80 & $4.79 \cdot 10^{-4}$ \\
\hline & & Aminoacyl-tRNA-synthetases & 15 & 39 & $7.46 \cdot 10^{-4}$ \\
\hline & & Energy & 58 & 360 & $1.69 \cdot 10^{-2}$ \\
\hline & & Pentose-phosphate pathway & 10 & 24 & $2.20 \cdot 10^{-2}$ \\
\hline & \multirow[t]{14}{*}{$\mathrm{GO}^{\mathrm{d}}$} & Translation & 117 & 345 & $6.31 \cdot 10^{-36}$ \\
\hline & & Cellular amino acid biosynthetic process & 44 & 101 & $1.10 \cdot 10^{-16}$ \\
\hline & & Ribosome biogenesis & 46 & 178 & $1.13 \cdot 10^{-7}$ \\
\hline & & Oxidation reduction & 60 & 270 & $1.24 \cdot 10^{-7}$ \\
\hline & & Metabolic process & 76 & 389 & $2.49 \cdot 10^{-7}$ \\
\hline & & Steroid biosynthetic process & 15 & 24 & $2.73 \cdot 10^{-7}$ \\
\hline & & Sterol biosynthetic process & 15 & 28 & $5.46 \cdot 10^{-6}$ \\
\hline & & Methionine biosynthetic process & 16 & 32 & $6.13 \cdot 10^{-6}$ \\
\hline & & Maturation of SSU-rRNA & 22 & 62 & $2.28 \cdot 10^{-5}$ \\
\hline & & Sulfate assimilation & 9 & 11 & $3.49 \cdot 10^{-5}$ \\
\hline & & rRNA processing & 43 & 195 & $8.44 \cdot 10^{-5}$ \\
\hline & & Methionine metabolic process & 10 & 15 & $1.36 \cdot 10^{-4}$ \\
\hline & & Lipid biosynthetic process & 18 & 52 & $7.16 \cdot 10^{-4}$ \\
\hline & & Ergosterol biosynthetic process & 7 & 9 & $2.66 \cdot 10^{-3}$ \\
\hline & \multirow[t]{6}{*}{$\mathrm{TF}^{\mathrm{d}}$} & FHL1 & 75 & 208 & $1.64 \cdot 10^{-24}$ \\
\hline & & RAP1 & 51 & 145 & $1.31 \cdot 10^{-15}$ \\
\hline & & SFP1 & 20 & 50 & $1.41 \cdot 10^{-6}$ \\
\hline & & GCN4 & 37 & 182 & $8.70 \cdot 10^{-4}$ \\
\hline & & HAP1 & 27 & 120 & $2.60 \cdot 10^{-3}$ \\
\hline & & MET32 & 9 & 24 & $4.06 \cdot 10^{-2}$ \\
\hline \multirow[t]{12}{*}{ Cluster 2} & \multirow[t]{7}{*}{ MIPS $^{d}$} & Unclassified proteins & 194 & 1140 & $4.13 \cdot 10^{-5}$ \\
\hline & & Oxidative stress response & 21 & 56 & $7.17 \cdot 10^{-4}$ \\
\hline & & Cell rescue, defense and virulence & 101 & 558 & $9.06 \cdot 10^{-3}$ \\
\hline & & Degradation of polyamines & 5 & 5 & $1.98 \cdot 10^{-2}$ \\
\hline & & Energy & 70 & 360 & $2.14 \cdot 10^{-2}$ \\
\hline & & Cellular communication & 50 & 239 & $4.60 \cdot 10^{-2}$ \\
\hline & & Cellular signalling & 44 & 202 & $4.71 \cdot 10^{-2}$ \\
\hline & \multirow[t]{5}{*}{$\mathrm{GO}^{\mathrm{d}}$} & Signal transduction & 24 & 74 & $4.64 \cdot 10^{-3}$ \\
\hline & & Protein amino acid phosphorylation & 36 & 141 & $1.11 \cdot 10^{-2}$ \\
\hline & & Proteasomal ubiquitin-dependent protein catabolic process & 9 & 16 & $3.95 \cdot 10^{-2}$ \\
\hline & & Oxidation reduction & 56 & 270 & $3.96 \cdot 10^{-2}$ \\
\hline & & Negative regulation of gluconeogenesis & 7 & 10 & $4.55 \cdot 10^{-2}$ \\
\hline
\end{tabular}


Table 1 continued

\begin{tabular}{llrrr}
\hline & Functional category & K $^{\mathbf{a}}$ & $\mathbf{N}^{\mathbf{b}}$ & $\mathbf{p ~ v a l u e}^{\mathbf{c}}$ \\
\hline TF $^{\mathbf{d}}$ & MSN2/MSN4 & 55 & 166 & $4.29 \cdot 10^{-11}$ \\
& SKN7 & 43 & 175 & $6.59 \cdot 10^{-4}$ \\
& YAP7 & 36 & 152 & $9.78 \cdot 10^{-3}$ \\
& CAD1 & 12 & 32 & $4.40 \cdot 10^{-2}$ \\
\hline
\end{tabular}

a Number of genes present in both the cluster and the functional category

b Total number of genes in the functional category

c A Bonferroni corrected $p$ value cut-off of 0.05 was used and $p$ values indicate the probability of finding the same number of genes in a random set

d Functional categories originate from the Munich Information Centre for Protein Sequences (MIPS), Gene Ontology (GO) or transcription factor binding datasets (TF) described in the "Methods" section

e MSN2/4 transcription factor dataset originates from [30]

conditions, functional categories related to protein synthesis were significantly enriched (Fig. 6).

\section{Extreme heat-shock tolerance of yeast cells grown in aerobic retentostats}

Studies in aerobic chemostats, anaerobic retentostats and aerobic stationary-phase batch cultures showed that slow growth of $S$. cerevisiae increases its stress tolerance, most often measured as its ability to survive exposure to high temperatures [13, 22, 34]. The aerobic chemostat cultures, grown at a specific growth rate of $0.025 \mathrm{~h}^{-1}$, which preceded the retentostat cultures were already remarkably heat-shock tolerant, with $50 \%$ of the population surviving a $115 \mathrm{~min}$ exposure to a temperature of $53{ }^{\circ} \mathrm{C}$ (Fig. 7a). After 10 days of retentostat cultivation, when the specific growth rate had decreased below $0.001 \mathrm{~h}^{-1}$, this $\mathrm{t}_{50}$ had increased to $4 \mathrm{~h}$. This $\mathrm{t}_{50}$ value is approximately fourfold higher than previously described for extremely heat-shock tolerant cultures, such as aerobic stationary-phase and anaerobic retentostat cultures (Fig. 7a). To the best of our knowledge, this heat-shock tolerance is the highest measured to date in S. cerevisiae.

In previous studies, a high heat-shock tolerance of $S$. cerevisiae was found to correlate with increased transcript levels of many known stress-responsive genes [13, 34]. Consistent with these earlier observations, transcript levels of $M s n 2 / 4$ gene targets, as well as genes that were previously shown to be responsive to environmental stresses (ESR induced: 110 out of 281 , p value $1.17 \cdot 10^{-30}$; ESR repressed: 170 out of 563 , p value $3.31 \cdot 10^{-48}$ ) or to heat shock in an Msn2/4-independent manner (125 out of 427 , $\mathrm{p}$ value $3.11 \cdot 10^{-21}$ ), correlated with specific growth rate and, therefore, with heat-shock tolerance in the aerobic retentostat cultures (Fig. 7b) [30, 35]. Heatshock proteins function as chaperones that prevent aggregation of thermally damaged proteins, unfold them, or mark them for degradation [29]. Of 76 genes known to encode heat-shock proteins, seven showed increased mRNA levels at near-zero growth rates (SSA3, HSP26, HSP42, XDJ1, CWC23, EUG1 and HSP60) [29]. Disaggregation and (re)folding activities of heat-shock proteins are ATP dependent and maintaining intracellular ATP levels has been shown to be crucial for heat-shock survival of stationary-phase batch cultures [34, 36]. High contents of the intracellular carbohydrate storage materials trehalose and glycogen ( $>10 \%$ of biomass dry-weight, Fig. 7c) may have contributed to the extreme heat-shock tolerance of yeast cells grown in aerobic retentostat cultures by supplying the ATP that is required to combat heat stress (Fig. 7c). In addition to intracellular trehalose concentrations, expression of the trehalose-metabolism related genes TPS1, TPS2, ATH1 and NTH1 increased substantially when retentostat cultures approached nearzero growth rates (Fig. 7c, d) [36, 37]. The strong increase of intracellular trehalose concentrations in the aerobic retentostat cultures represents a marked difference with published data on anaerobic retentostats, in which intracellular trehalose levels were low and glycogen was the predominant storage carbohydrate [14].

\section{Aerobic retentostat cultures retain a high glycolytic capacity at near-zero growth rates}

Glycolysis, together with glucose transport, pyruvate decarboxylase and alcohol dehydrogenase, represents the pathway for alcoholic fermentation in S. cerevisiae. Respiratory cultures of this yeast maintain a high capacity for fermentative metabolism (fermentative capacity), which allows $S$. cerevisiae to rapidly increase its glycolytic flux in response to, for example, oxygen depletion and/or exposure to high sugar concentrations [38-40]. In aerobic glucose-limited chemostat cultures of the $S$. cerevisiae CEN.PK113-7D strain, fermentative capacity is essentially growth-rate independent at specific growth rates between 0.05 and $0.3 \mathrm{~h}^{-1}$, [28]. The fermentative 


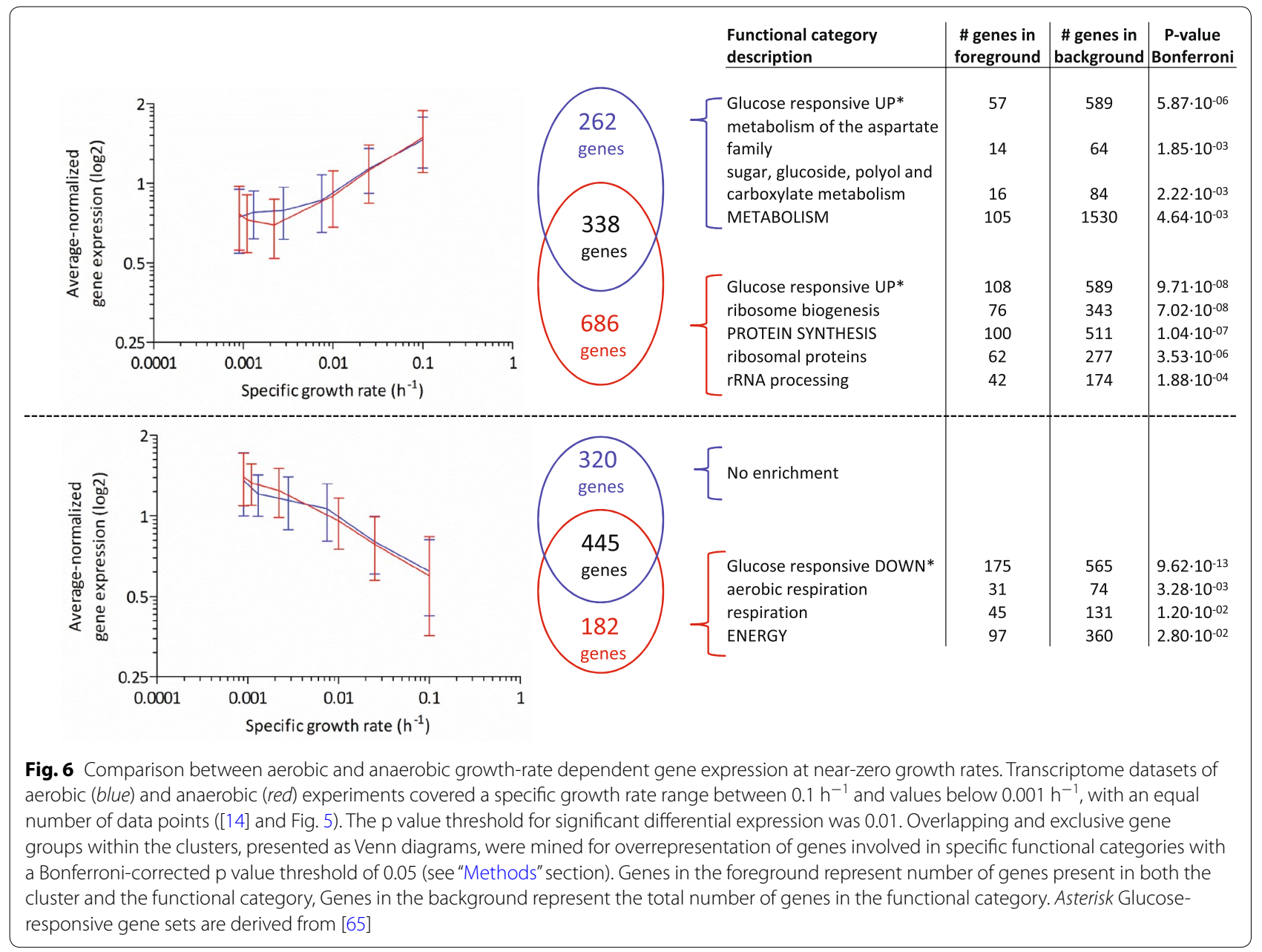

capacity of $7.5 \mathrm{mmol}$ ethanol $\mathrm{g}_{\mathrm{X}}{ }^{-1} \mathrm{~h}^{-1}$ measured in the aerobic chemostats $\left(D=0.025 \mathrm{~h}^{-1}\right.$, Fig. 8a), matched well with the fermentative capacity found previously at these higher specific growth rates [28]. After 18 days of aerobic retentostat cultivation, a significantly lower ( $\mathrm{p}$ value $<0.05$ ) fermentative capacity of $4.5 \mathrm{mmol}$ ethanol $g_{\mathrm{X}}^{-1} \mathrm{~h}^{-1}$ was measured (Fig. 8a). The corresponding glucose-consumption rate was 45 -fold higher than the specific rate of glucose consumption measured in the retentostat at this time point.

The decrease of the fermentative capacity in the aerobic retentostats coincided with a decrease of the transcript levels of a subset of glycolytic genes, some of which encoded major isoforms of glycolytic enzymes [41]. Expression levels of $H X K 2$ encoding hexokinase 2, first step in glycolysis, $F B A 1$ encoding the single fructose-bisphosphate aldolase, $P G K 1$ encoding the single phosphoglycerate kinase, GPM1 encoding the major phosphoglycerate mutase, ENO1 and ENO2 paralogs encoding the two yeast enolases, $P Y K 1$ also known as $C D C 19$, encoding the major pyruvate kinase, last step in glycolysis, and PDC1 encoding pyruvate decarboxylase 1 responsible for the first step in the fermentative pathway leading to ethanol, were stable during the initial phase of the retentostat cultures, but significantly and substantially decreased at growth rates below $0.002 \mathrm{~h}^{-1}$ (Fig. 8b). Pair-wise comparison of transcriptome data for day 0 and day 16 of the retentostats (corresponding to specific growth rates of 0.025 and $0.0009 \mathrm{~h}^{-1}$, respectively) showed at least a twofold difference in expression levels of HXK2, PGK1, GPM1, ENO2 and PDC1. Overrepresentation of binding sites for Rap1/Gcr1 in their promoter regions suggests that these transcription factors may be involved in their transcriptional regulation at near-zero growth rates. This hypothesis is further supported by the observation that 51 of the 145 gene targets of the transcription factor Rap1 were part of cluster 1 (Fig. 4, Table 1). While we cannot exclude that decreased glucose transport was also involved in the reduction of fermentative capacity, no difference was observed in $H X T$ gene expression at near-zero growth rates. 

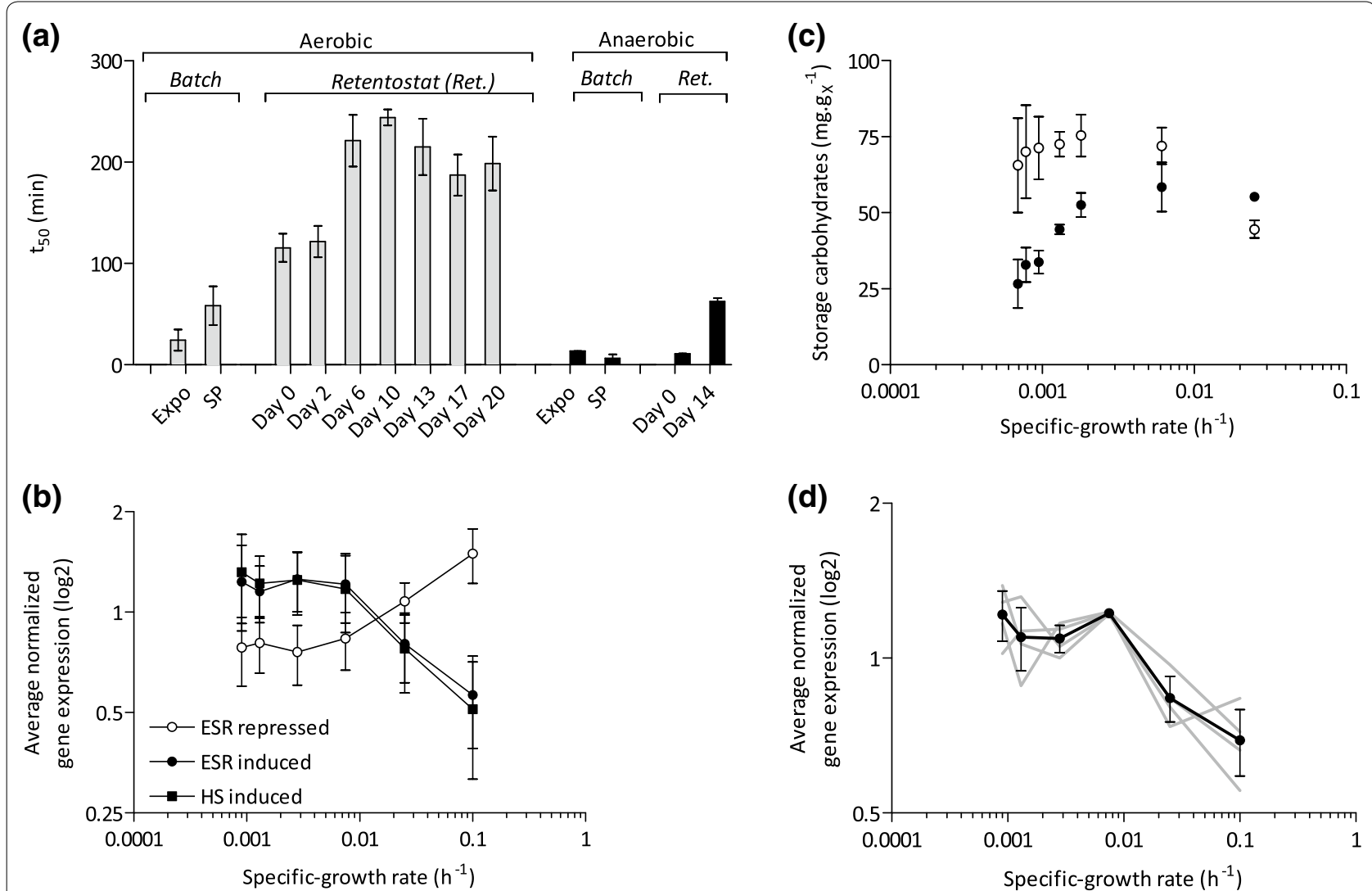

Fig. 7 Heat-shock tolerance of aerobic and anaerobic retentostat and batch cultures. a Data on heat shock tolerance of anaerobic retentostat cultures and from batch cultures are taken from previous studies [13, 34]. Batch cultures were characterized during the exponential growth phase (expo) and after ca. $2 \mathrm{~h}$ in stationary phase (SP) [34]. $\mathrm{t}_{50}$ represents incubation time at $53^{\circ} \mathrm{C}$ at which $50 \%$ of the initial viable cell population was still alive. $\mathbf{b}$ Transcript levels of genes that exhibit a significant growth-rate dependent expression in retentostat, and are also known to respond to environmental stress and heat shock according to [30,35]. c Cellular contents of trehalose (open symbols) and glycogen (closed symbols) during prolonged retentostat cultivation. d Average-normalized expression profiles of genes involved in trehalose metabolism (see "Methods" section)
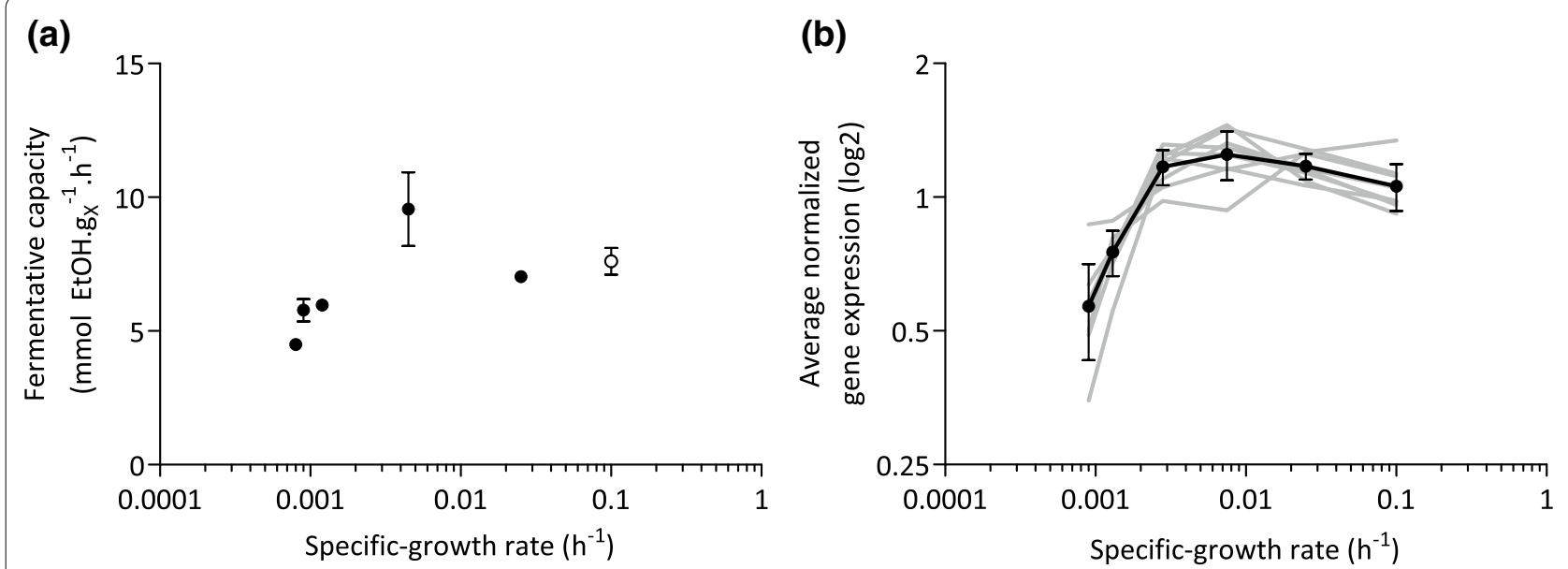

Fig. 8 Fermentative capacity and expression levels of glycolytic genes in S. cerevisiae at near-zero growth rates. a Fermentative capacity, measured off-line as the specific rate of ethanol formation upon exposure of anaerobic cell suspensions to excess glucose. Fermentative capacity assays were performed on independent duplicate retentostat cultures, sampled at different time points. The open symbol corresponds to data from [28]. b Log2 average-normalized gene expression of HXK2, FBA1, PGK1, GPM1, ENO1, ENO2, PYK1, and PDC1 during retentostat cultivation, plotted as a function of specific growth rate (see "Methods" section) 


\section{Discussion}

\section{Estimation of maintenance-energy requirements} from aerobic retentostats

Initially developed by microbial ecologists to explore the 'twilight zone' between exponential growth and starvation [17, 19-21, 42, 43], the retentostat has recently seen a revival in studies on industrial microorganisms [18]. A key advantage of retentostat cultivation for applicationinspired research is that it enables an accurate, quantitative estimation of microbial maintenance-energy requirements [18]. The conventional method for determining $\mathrm{m}_{\mathrm{S}}$ does not measure, but estimates the specific rate of energy-substrate consumption in non-growing cultures, based on extrapolation of measurements on chemostat cultures that are actively growing (often at specific growth rates of $0.05 \mathrm{~h}^{-1}$ and above). Since, at these specific growth rates, substrate consumption for maintenance is relatively small as compared to the overall consumption rate of the energy substrate, $\mathrm{m}_{\mathrm{S}}$ values calculated via this procedure are sensitive to small measurement errors [3, 18]. Moreover, chemostat-based estimation of $m_{\mathrm{S}}$ is based on the assumption that this parameter is growth-rate independent. Studies on several prokaryotes have shown that this assumption is not always valid and that, at extremely slow growth rates, several bacteria downregulate ATP turnover and thereby reduce substrate consumption for maintenance [20,44].

Even at extremely low specific growth rates, the energetics of aerobic, glucose-limited retentostat cultures of $S$. cerevisiae could be adequately described with a growth-rate independent $m_{S}$. The same conclusion was drawn earlier for anaerobic, glucose-limited retentostat cultures of this yeast [12]. The value of $\mathrm{m}_{\mathrm{S}}$ estimated from the aerobic retentostat cultures was $0.039 \mathrm{mmol}$ glucose $g_{X}^{-1} h^{-1}$. There are surprisingly few, invariably chemostat-based, estimates of the $\mathrm{m}_{\mathrm{S}}$ of aerobically grown $S$. cerevisiae. Four decades ago, Rogers and Stewart [45] calculated an $\mathrm{m}_{\mathrm{S}}$ of $0.07 \mathrm{mmol}$ glucose $\mathrm{g}_{\mathrm{X}}^{-1} \mathrm{~h}^{-1}$ based on aerobic chemostat cultures of a diploid $S$. cerevisiae strain, grown at pH 5.5 on a complex medium. This value is $75 \%$ higher than the $\mathrm{m}_{\mathrm{S}}$ found in the present study. Recently, based on aerobic chemostat cultures of the same haploid $S$. cerevisiae strain that is used in the present study, grown at $\mathrm{pH} 5.5$, we estimated an $\mathrm{m}_{\mathrm{S}}$ that was even 2.5fold higher than calculated from the aerobic retentostats [3]. It should, however, be noted that the latter study used a growth medium that contained high concentrations of copper, which may have negatively affected cellular energetics.

Based on an assumed P/O ratio of 1.0 [23, 29, 46, 47], the maintenance requirement for ATP $\left(\mathrm{m}_{\mathrm{ATP}}\right)$ estimated from the aerobic retentostat cultures was $0.63 \mathrm{mmol}$ ATP $g_{X}^{-1} h^{-1}$, a value $35 \%$ lower than previously estimated based on anaerobic retentostat cultures of the same $S$. cerevisiae strain [12]. One possible explanation for this difference is that anaerobic growth indeed results in a higher $m_{S}$, for instance as a result of increasing proton leakage across membranes due to the presence of the fermentation products ethanol and acetic acid. Additionally, the anaerobic growth factor oleic acid, which is added to anaerobic chemostat media as the oleate ester Tween-80, has been shown to negatively affect growth energetics [25]. Alternatively, the assumed $\mathrm{P} / \mathrm{O}$ value of 1.0 might be wrong. However, if this were the sole reason for the observed difference, the actual $\mathrm{P} / \mathrm{O}$ ratio would have to be close to 1.7 , which falls outside the range of estimates for this parameter from several quantitative physiological studies on $S$. cerevisiae [23, 29, 46, 47]. The lower $\mathrm{m}_{\text {ATP }}$ under aerobic conditions, makes it unlikely that the presence of oxygen or generation of ROS in respiration increases maintenance-energy requirements.

Maintenance-energy requirements are well known to depend on growth conditions, for example on the presence of weak organic acids [48-50], and may additionally be strain dependent. The present study demonstrates that retentostat cultivation offers a robust way to estimate $m_{S}$. The large impact of this parameter on the performance of large-scale industrial fed-batch processes provides a strong impetus for using this, somewhat technically demanding, approach for determining and comparing maintenance-energy requirements of different production hosts under carefully controlled, industrially relevant experimental conditions.

\section{Extreme heat-shock tolerance of aerobic retentostat cultures}

In industrial processes, yeast cells face a variety of stresses, including high concentrations of $\mathrm{CO}_{2}$ and other products, inhibitors in low-grade media, fluctuations in nutrient availability (e.g. during biomass recycling and 'repitching' in beer fermentation) and high as well as low temperatures $[51,52]$. Here we show that aerobic retentostat cultures of $S$. cerevisiae grown at near-zero growth rates acquire an extreme resilience to heat shock. We recently reported that stationary-phase, glucose-grown aerobic batch cultures of $S$. cerevisiae are much more heat-shock tolerant than the corresponding anaerobic cultures [34]. This difference was tentatively attributed to the much faster transition from exponential growth to nutrient depletion in anaerobic batch cultures, which do not exhibit the second, slow growth phase on ethanol that is characteristic for aerobic glucose-grown batch cultures of S. cerevisiae. The hypothesis that this fast transition prevented a full induction of heat-shock tolerance was consistent with the earlier observation that anaerobic retentostat cultures, which undergo a slow transition to near-zero growth rates, 
exhibit a much higher heat-shock tolerance than anaerobic stationary-phase batch cultures [34]. The present study shows that, despite a very similar 'conditioning', the heatshock tolerance of aerobic retentostat cultures is much more pronounced than in anaerobic retentostats (four to fivefold higher $t_{50}$, Fig. 7a). Indeed, to our knowledge, the heat-shock tolerance of the aerobic retentostat cultures is the highest reported to date for S. cerevisiae. These observations indicated that a smooth transition from exponential growth to (near-)zero growth in aerobic cultures provides an optimal conditioning for heat-shock tolerance in this yeast. Further research is required to assess whether this conclusion can be extended to include conditioning for other industrially relevant stresses, such as freezing/ drying, osmotic stress and oxidative stress.

Intracellular concentrations of trehalose and regulation of genes involved in its metabolism showed a remarkable correlation with the different levels of heat-shock tolerance in aerobic retentostats. Trehalose can act as an energy reserve, and has also been proposed to be directly involved in heat shock resistance [53-55]. However, recent evidence suggests that secondary, so called 'moonlighting' functions of the trehalose-6-phosphate synthase Tps1, rather than trehalose itself, contribute to cell integrity during heat shock [36]. Additionally, different expression levels of other stress-induced proteins and different membrane composition, resulting from the inability of anaerobic cultures to synthetize unsaturated fatty acid and sterols, may contribute to the different heat-shock tolerance of aerobic and anaerobic $S$. cerevisiae cultures $[25,56,57]$.

\section{S. cerevisiae down-regulates glycolytic gene expression but maintains a high fermentative capacity at near-zero growth}

Protein synthesis is the single most ATP-intensive process in living cells [58], and especially proteins with relatively high expression levels and short turnover times are expected to represent a significant metabolic burden to cells grown under severely calorie-restricted retentostat cultivation regimes. In actively growing $S$. cerevisiae cultures, glycolytic enzymes make up a significant fraction of the total cellular protein [59]. The half-life of most glycolytic proteins in S. cerevisiae grown in glucose-excess conditions range between 5 and $20 \mathrm{~h}$, excluding Tdh1, Tdh2, Gpm2, and Eno1, for which half-lives of over $100 \mathrm{~h}$ have been determined [60]. These reported half-lives are much lower than the amount of time that the cells reside in retentostat; protein turnover of glycolytic proteins could therefore significantly contribute to the energy requirements of $S$. cerevisiae at near-zero growth. Under many conditions, this yeast exhibits a large overcapacity of the glycolytic pathway. Indeed, a substantial loss of fermentative capacity has previously been observed during prolonged cultivation of $S$. cerevisiae in aerobic, glucose-limited chemostat cultures (50\% after 100 generations) [61]. This loss was attributed to mutations that reduced the metabolic burden of synthesizing large amounts of glycolytic proteins. Although retentostatgrown cells retained a high glycolytic capacity, this decreased by ca. $40 \%$ at extremely low specific growth rates. It is, however, unlikely that evolutionary adaptation caused this reduction in glycolytic capacity, since the average number of generations in the retentostat experiments was approximately three as a consequence of the biomass retention. Instead, the reduced mRNA levels of several glycolytic genes suggest a transcriptional downregulation of this key pathway at extremely low growth rates. Furthermore, glycolytic genes $P G K 1$ and $P Y K 1$ that are considered to be constitutively expressed at high levels [62], displayed ca. twofold reduced transcript levels at near-zero growth (Fig. 8), and shows that glycolytic promoters for the expression of (heterologous) proteins should be carefully selected.

\section{Impact of oxygen availability on transcriptional reprogramming at near-zero growth rates}

The specific growth rate profiles and experimental conditions employed in the aerobic retentostat cultures very strongly resembled those applied in a previous study on anaerobic retentostats of the same $S$. cerevisiae strain. Gene sets that showed a transcriptional response in these retentostat experiments showed a strong overrepresentation of growth-rate responsive genes identified by Fazio et al. [63]. These authors used chemostats, grown at specific growth rates of $0.03 \mathrm{~h}^{-1}$ and higher, to explore transcriptional responses under different aerobic and anaerobic nutrient-limitation. Of the set of 268 growthrate-responsive genes identified in their study, 115 genes were also found to show growth-rate dependent expression at the very low specific growth rates studied in the aerobic and anaerobic retentostats (Additional file 3). Despite this clear overlap in transcriptional responses, the number of genes that showed a transcriptional response to the shift to near-zero growth rates was twofold higher in the anaerobic retentostats than in the aerobic retentostats. As discussed above, ATP yields from respiratory and fermentative glucose dissimilation differ by a factor of approximately 8 . As a consequence, at any specific growth rate, specific rates of glucose consumption $\left(\mathrm{q}_{\mathrm{S}}\right)$ in anaerobic glucose-limited cultures are higher than in the corresponding aerobic cultures. For example, at a specific growth rate of $0.025 \mathrm{~h}^{-1}$, the $\mathrm{q}_{\mathrm{S}}$ in anaerobic glucose-limited chemostat cultures $\left(2.3 \mathrm{mmol} \mathrm{g}_{\mathrm{x}}^{-1} \mathrm{~h}^{-1}\right.$ (Additional file 4, [12]), was ca. eightfold higher than in the corresponding aerobic chemostat cultures 
[0.3 mmol g $\mathrm{x}^{-1} \mathrm{~h}^{-1}$ (Additional file 4)]. Simple monod kinetics [64] predict that this difference should also be reflected in the concentration of the growth-limiting nutrient. Indeed, residual glucose concentrations in these anaerobic and aerobic cultures were 0.3 and $0.07 \mathrm{mM}$, respectively (Additional file 4 and [14]). The consequence of these differences is that aerobic and anaerobic retentostat cultures operate in a different range of residual glucose concentrations. Concomitantly, a set of previously identified glucose-responsive transcripts were specifically overrepresented under anaerobiosis among genes which were transcriptionally up and down regulated with specific growth rate in retentostat cultures (Fig. 6) [65]. This comparison identifies differences in glucose concentration as a major cause of the different transcriptome profiles of aerobic and anaerobic retentostat cultures.

\section{Conclusion}

Glucose-feeding regimes of retentostat cultures were optimized by model simulations to enable a first characterization of glucose-limited, aerobic cultures of $S$. cerevisiae during a smooth transition to extremely low specific growth rates. Quantitative analysis of these retentostats enabled the most accurate estimation to date of the growth-rate-independent maintenance-energy requirement of this yeast. Aerobic, glucose-limited retentostat cultures of $S$. cerevisiae showed a high viability, an extremely high heat-shock tolerance and retained an overcapacity of the fermentative pathway, thus illustrating the potential of this yeast to be developed for robust product formation in the absence of growth. This study shows that retentostat cultures, although technically demanding, offer unique possibilities for quantitative analysis of industrially relevant aspects of microbial physiology.

\section{Methods}

\section{Yeast strain and shake-flask cultivation}

The prototrophic strain S. cerevisiae CEN.PK113-7D (MATa, MAL2-8c, SUC2; [66, 67]) was used in this study. Stock cultures were grown in $500 \mathrm{~mL}$ shake flasks containing $100 \mathrm{~mL}$ YPD medium $\left(10 \mathrm{~g} \mathrm{~L}^{-1}\right.$ Bacto yeast extract, $20 \mathrm{~g} \mathrm{~L}^{-1}$ Bacto peptone and $20 \mathrm{~g} \mathrm{~L}^{-1} \mathrm{D}$-glucose). After addition of glycerol $(20 \% \mathrm{v} / \mathrm{v})$ to early stationaryphase cultures, $2 \mathrm{~mL}$ aliquots were stored at $-80{ }^{\circ} \mathrm{C}$. Shake-flask precultures for chemostat experiments were grown in $500 \mathrm{~mL}$ shake flasks containing $100 \mathrm{~mL}$ of synthetic medium, set to $\mathrm{pH} 6.0$ with $2 \mathrm{M} \mathrm{KOH}$ prior to autoclaving and supplemented with $20 \mathrm{~g} \mathrm{~L}^{-1} \mathrm{D}$-glucose [49]. These shake-flask cultures were inoculated with $2 \mathrm{~mL}$ of frozen stock culture and incubated in an orbital shaker at $200 \mathrm{rpm}$ and at $30^{\circ} \mathrm{C}$.

\section{Chemostat cultivation}

Chemostat cultivation was performed in 2-liter bioreactors (Applikon, Delft, the Netherlands) equipped with a level sensor to maintain a constant working volume of $1.4 \mathrm{~L}$. The culture temperature was controlled at $30{ }^{\circ} \mathrm{C}$ and the dilution rate was set at $0.025 \mathrm{~h}^{-1}$ by controlling the medium inflow rate. Cultures were grown on synthetic medium, prepared as described previously [49] but with the following modifications: the glucose concentration was increased to $20 \mathrm{~g} \mathrm{~L}^{-1}$ glucose $\left(\mathrm{C}_{\mathrm{S}, \mathrm{MC}}\right)$, the amount of trace-element and vitamin solutions were increased to 1.5 and $2 \mathrm{~mL} \mathrm{~L}^{-1}$ respectively [49], and $0.25 \mathrm{~g} \mathrm{~L}^{-1}$ Pluronic $6100 \mathrm{PE}$ antifoaming agent (BASF, Ludwigshafen, Germany) was used. Fresh medium was supplied to the bioreactor from a 3-liter stirred mixing vessel (Applikon, Delft, The Netherlands) whose working volume $\left(V_{S}\right)$ of $1.2 \mathrm{~L}$ was controlled by a level sensor and which was stirred continuously at $500 \mathrm{rpm}$. The mixing vessel was equipped with a sampling port. Medium was added to the mixing reactor by automatic addition from a medium reservoir, with a flow rate $\left(\phi_{\mathrm{V}}\right)$ of $35 \mathrm{~mL} \mathrm{~h}^{-1}$ corresponding to the dilution rate in the bioreactor. Cultures were sparged with air $(0.5 \mathrm{vvm})$ and stirred at $800 \mathrm{rpm}$. Culture $\mathrm{pH}$ was kept constant at 5.0 by automatic addition of $10 \% \mathrm{NH}_{4} \mathrm{OH}$. Chemostat cultures were assumed to be in steady state when, after at least 6 volume changes, culture dry weight and the specific carbon dioxide production rates changed by less than $3 \%$ over 2 consecutive volume changes. Steady-state carbon recoveries of chemostat cultures included in this study were above $98 \%$. Chemostat experiments performed at a dilution rate of $0.10 \mathrm{~h}^{-1}$ were performed as described above, with the following modifications: cultures were grown on synthetic medium [49] without modifications, with $7.5 \mathrm{~g} \mathrm{~L}^{-1}$ glucose, $1 \mathrm{~mL} \mathrm{~L}^{-1}$ trace elements solution, and $1 \mathrm{~mL} \mathrm{~L}^{-1}$ vitamin stock solution.

\section{Retentostat}

After reaching a steady-state in chemostat cultures, the retentostat phase was started by switching the reactor effluent to an outflow port equipped with an autoclavable Applisense filter assembly (Applikon), consisting of a hydrophobic polypropylene filter with a pore size of $0.22 \mu \mathrm{m}$ and a stainless steel hollow filter support. Prior to autoclaving, the filter was wetted by overnight incubation in $96 \%$ ethanol, and subsequently rinsed with a phosphate buffer saline solution (containing per $1 \mathrm{~L}$ demi-water: $8 \mathrm{~g} \mathrm{NaCl}, 0.2 \mathrm{~g} \mathrm{KCl}, 1.44 \mathrm{~g} \mathrm{Na}_{2} \mathrm{HPO}_{4}, 0.24 \mathrm{~g}$ $\mathrm{KH}_{2} \mathrm{PO}_{4}$, and $\mathrm{HCl}$ to adjust the final $\mathrm{pH}$ to 7.4). To control biomass accumulation, the medium reservoir connected to the mixing vessel (see above) was exchanged 
for a reservoir containing standard synthetic medium [49] supplemented with $7.5 \mathrm{~g} \mathrm{~L}^{-1}$ glucose $\left(\mathrm{C}_{\mathrm{S}, \mathrm{MR}}\right)$ and $0.25 \mathrm{~g} \mathrm{~L}^{-1}$ pluronic $6100 \mathrm{PE}$ antifoam. Consequently, the concentration of growth-limiting substrate glucose entering the bioreactor $\left[\mathrm{C}_{\mathrm{S}, \text { in }}\right.$ in $\left.\left(\mathrm{g} \mathrm{L}^{-1}\right)\right]$ decreased over time $[t$ in (h)] according to Eq. 3.

$$
C_{S, i n}=\left(C_{S, M C}-C_{S, M R}\right) \cdot e^{\frac{-\phi_{V} t}{V_{S}}}+C_{S, M R}
$$

In this equation, $\mathrm{C}_{\mathrm{S}, \mathrm{MC}}$ and $\mathrm{C}_{\mathrm{S}, \mathrm{MR}}$ correspond to the glucose concentrations in the medium entering the mixing vessel during the chemostat and the retentostat phase respectively. During retentostat cultivaton, culture $\mathrm{pH}$ was controlled by automatic addition of $2 \mathrm{M} \mathrm{KOH}$. Sampling frequency and sample volume were minimized to limit the impact of sampling on biomass accumulation inside the reactor. Culture purity was routinely checked by microscopy and by plating on synthetic medium agar containing $20 \mathrm{~g} \mathrm{~L}^{-1}$ glucose and $20 \mathrm{mM} \mathrm{LiCl} \mathrm{[68].} \mathrm{Full}$ biomass retention was confirmed by plating filtered effluent on YPD containing $2 \%(\mathrm{w} / \mathrm{v})$ agar.

\section{Predicting retentostat growth kinetics}

Operational conditions to enable a smooth transition of the retentostat cultures to near-zero growth rates, were defined with a mathematical model that simulates growth kinetics of yeast during aerobic retentostat cultivation (See Additional files 5, 6, 7). Essentially, the mass balance equation for biomass (Eq. 4) was solved using MATLAB ${ }^{\circledR}$ ode45 solver, by incorporating the substrate mass balance (Eq. 5), with the Pirt relation [69] (Eq. 1; Fig. 1e).

$$
\begin{aligned}
& \frac{d C_{X}}{d t}=\mu C_{X} \\
& \frac{d C_{S}}{d t}=\frac{\phi_{V}}{V}\left(C_{S, \text { in }}-C_{S}\right)-q_{S} C_{X}
\end{aligned}
$$

In these equations, $C_{X}\left(g^{-1}\right)$ is the biomass concentration in the retentostat, $\mu\left(\mathrm{h}^{-1}\right)$ is the specific growth rate, $C_{S}\left(g^{-1}\right)$ is the residual substrate concentration, $\mathrm{C}_{\mathrm{S}, \text { in }}\left(\mathrm{g} \mathrm{L}^{-1}\right)$ is the substrate concentration in the feed, $\frac{\phi \mathrm{V}}{\mathrm{V}}\left(\mathrm{h}^{-1}\right)$ is the dilution rate, and $\mathrm{q}_{\mathrm{S}}\left(\mathrm{g} \mathrm{g}_{\mathrm{X}}^{-1} \mathrm{~h}^{-1}\right)$ is the biomass-specific glucose consumption rate. The specific substrate consumption rate can be described by the Pirt relation (Eq. 1), in which $\mathrm{Y}_{\mathrm{X} / \mathrm{S}}^{\max }\left[\mathrm{g} \mathrm{g}^{-1}\right]$ is the maximum biomass yield on glucose, and $\mathrm{m}_{\mathrm{S}}\left(\mathrm{g} \mathrm{g}_{\mathrm{X}}^{-1} \mathrm{~h}^{-1}\right)$ is the maintenance coefficient. Because retentostats were glucose limited and $C_{S, \text { in }} \gg C_{S}$, the glucose concentration in the retentostat was assumed to be in a pseudo-steady state such that $\mathrm{dC}_{\mathrm{S}} / \mathrm{dt} \approx 0$.

To run simulations, the model required inputs for variables $\mathrm{V}$ (bioreactor volume) (L), $\phi_{\mathrm{V}}$ (flow rate) $\left(\mathrm{L} \mathrm{h}^{-1}\right)$,
$\mathrm{C}_{\mathrm{S}, \mathrm{MC}}\left(\mathrm{g} \mathrm{L}^{-1}\right), \mathrm{C}_{\mathrm{S}, \mathrm{MR}}\left(\mathrm{g} \mathrm{L}^{-1}\right)$, and $\mathrm{V}_{\mathrm{S}}(\mathrm{L})$, and generated time-dependent profiles for biomass accumulation, glucose concentration in the feed, specific glucose consumption rates, and specific growth rates for a range of $m_{S}$ values. The final operational conditions chosen for the retentostat experiments are indicated in Fig. 2.

\section{Regression analysis of biomass accumulation in retentostat} The maintenance-energy requirements and biomassspecific death rate of $S$. cerevisiae in aerobic retentostat were estimated from a least-squares regression analysis of data points for the biomass concentration (dry-weight) and the viable biomass concentration over time, using a MATLAB model (see Additional files 8, 9, 10, 11, 12). From these parameters, the specific growth rate and substrate consumption rates were derived. The curve shape was determined by the solution of the following ordinary differential equations with the smallest sum of square errors:

$$
\begin{aligned}
& \frac{d C_{X_{-} V}}{d t}=\mu C_{X_{-} V}-k_{d} C_{X_{-} V} \\
& \frac{d C_{X_{-}}}{d t}=k_{d} C_{X_{-} V} \\
& \frac{d C_{S}}{d t}=\frac{\phi_{V}}{V}\left(C_{S, i n}-C_{S}\right)-q_{S} C_{X_{-} V}
\end{aligned}
$$

In these equations, $C_{X_{-}}$is the viable biomass concentration $\left(\mathrm{g} \mathrm{L}^{-1}\right), \mathrm{k}_{\mathrm{d}}$ is the death rate $\left(\mathrm{h}^{-1}\right)$. Equation 1 was used to define the specific substrate consumption rate $\left(\mathrm{q}_{\mathrm{s}}\right)$.

The model required input for the biomass concentrations measured at different time points, and the following variables: $\mathrm{V}(\mathrm{L}), \phi_{\mathrm{V}}\left(\mathrm{L} \mathrm{h}^{-1}\right), \mathrm{C}_{\mathrm{S}, \mathrm{MC}}\left(\mathrm{g} \mathrm{L}^{-1}\right), \mathrm{C}_{\mathrm{S}, \mathrm{MR}}\left(\mathrm{g} \mathrm{L}^{-1}\right)$, $\mathrm{V}_{\mathrm{S}}(\mathrm{L})$ and $\mathrm{Y}_{\mathrm{X} / \mathrm{S}}^{\max }$. A value for $\mathrm{m}_{\mathrm{S}}$ was approximated using parameter estimation. The time-dependent change of $\mathrm{q}_{\mathrm{S}}$ and $\mu$ during the course of the retentostat followed from the regression analysis (see Additional files 8, 9, 10, 11, 12). To respect small differences in operational variables per experiment, regression analyses were performed separately on each independent retentostat experiment.

\section{Determination of substrate, metabolites and biomass concentration}

Prior to culture dry weight assays, retentostat samples were diluted in demineralized water. Culture dry weight was measured by filtering exactly $10 \mathrm{~mL}$ of an appropriate dilution of culture broth over pre-dried and preweighed membrane filters (pore size $0.45 \mu \mathrm{m}$, Gelman Science), which were then washed with demineralized water, dried in a microwave oven $(20 \mathrm{~min}, 350 \mathrm{~W})$ and 
reweighed. Supernatants were obtained by centrifugation of culture samples ( $3 \mathrm{~min}$ at $16,000 \times g$ ) and analysed by high-performance liquid chromatography (HPLC) analysis on a Agilent 1100 HPLC (Agilent Technologies, Santa Clara, CA, USA), equipped with an Aminex HPX-87H ion-exchange column (BioRad, Veenendaal, The Netherlands), operated with $5 \mathrm{mM} \mathrm{H}_{2} \mathrm{SO}_{4}$ as the mobile phase at a flow rate of $0.6 \mathrm{~mL} \mathrm{~min}{ }^{-1}$ and at $60{ }^{\circ} \mathrm{C}$. Detection was by means of a dual-wavelength absorbance detector (Agilent G1314A) and a refractive-index detector (Agilent G1362A). Residual glucose concentrations in chemostat and retentostat cultures were analysed by HPLC after rapid quenching of culture samples with cold steel beads [70].

\section{Gas analysis}

The exhaust gas from chemostat cultures was cooled with a condenser $\left(2{ }^{\circ} \mathrm{C}\right)$ and dried with a PermaPure Dryer (model MD 110-8P-4; Inacom Instruments, Veenendaal, the Netherlands) prior to online analysis of carbon dioxide and oxygen with a Rosemount NGA 2000 Analyser (Baar, Switzerland).

\section{Viability}

Small aliquots of culture broth $(<1 \mathrm{~mL})$ were sampled in a $10 \mathrm{mM} \mathrm{Na-Hepes} \mathrm{buffer} \mathrm{(pH} \mathrm{7.2)} \mathrm{with} 2 \%$ glucose. Cell numbers were determined with a Coulter counter using a $50 \mu \mathrm{m}$ orifice (Multisizer II, Beckman, Fullerton, $\mathrm{CA}$ ). Colony-forming units (CFU) in culture samples were quantified by triplicate plating of 10-fold dilution series in $0.1 \%$ peptone on $2 \%$ YPD agar plates. At least 150 colonies were counted after 2 days of incubation at $30{ }^{\circ} \mathrm{C}$ to calculate CFU. Viability was then calculated by comparing CFU counts with total cell counts. Additionally culture viability was assayed by propidium iodide (PI) staining (Invitrogen, Carlsbad, CA) by counting 10,000 cells on a Cell Lab Quanta SC MPL flow cytometer (Beckman Coulter, Woerden, Netherlands) as described previously [13]. PI intercalates with DNA in cells with a compromised cell membrane, causing a red fluorescence.

\section{Heat shock resistance assays}

Samples from retentostat cultures were added to Isoton II diluent (Beckman Coulter, Woerden, Netherlands), pre-heated at $53{ }^{\circ} \mathrm{C}$, to a final concentration of $10^{7}$ cells $\mathrm{mL}^{-1}$, and incubated at $53{ }^{\circ} \mathrm{C}$ for at least $200 \mathrm{~min}$. Loss of viability was monitored by sampling at $20 \mathrm{~min}$ intervals. Samples were immediately cooled on ice and subsequently stained with PI and analysed by flow cytometry as described above. Heat-shock resistance was represented by $\mathrm{t}_{50}$, the incubation time at $53{ }^{\circ} \mathrm{C}$ that lead to a $50 \%$ decrease in viability. To calculate $t_{50}$, survival curves were fitted with a sigmoidal dose-response curve in Graphpad ${ }^{\circledR}$ Prism, version 4.03.

\section{Glycogen and trehalose assays}

$1 \mathrm{~mL}$ broth was sampled from the retentostat or chemostat and immediately added to $5 \mathrm{~mL}$ of cold methanol $\left(-40{ }^{\circ} \mathrm{C}\right)$, mixed and centrifuged $\left(4400 \times g,-19{ }^{\circ} \mathrm{C}\right.$, $5 \mathrm{~min})$. The supernatant was decanted and pellets were resuspended in $5 \mathrm{~mL}$ cold methanol, pelleted again and stored at $-80{ }^{\circ} \mathrm{C}$. Pellets were then resuspended and diluted in $0.25 \mathrm{M} \mathrm{Na}_{2} \mathrm{CO}_{3}$, and further processed as previously described [71]. Trehalose was directly measured by HPLC. Glucose released from glycogen was measured by HPLC after overnight incubation of samples at $57{ }^{\circ} \mathrm{C}$ with a-amyloglucosidase (from Aspergillus niger, SigmaAldrich, Zwijndrecht, Netherlands).

\section{Fermentative capacity assays}

Samples containing exactly $100 \mathrm{mg}$ dry weight of biomass from retentostat cultures were harvested by centrifugation at $5000 \times g$ for $5 \mathrm{~min}$, washed once, and resuspended in $10 \mathrm{~mL}$ fivefold concentrated synthetic medium ( $\mathrm{pH} \mathrm{6,} \mathrm{[49]).} \mathrm{Subsequently,} \mathrm{these} \mathrm{cell} \mathrm{suspen-}$ sions were introduced into a $100 \mathrm{~mL}$ reaction vessel maintained at $30{ }^{\circ} \mathrm{C}$, which was kept anaerobic with

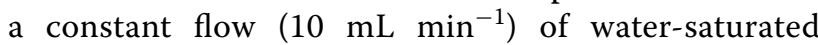
$\mathrm{CO}_{2}$. After addition of $40 \mathrm{~mL}$ demineralized water and $10 \mathrm{~min}$ of pre-incubation, $10 \mathrm{~mL}$ glucose solution (100 $\left.\mathrm{g} \mathrm{L}^{-1}\right)$ was added, and $1 \mathrm{~mL}$ samples were taken at 5 min intervals. After centrifugation, ethanol concentrations in supernatants were determined by HPLC. Fermentative capacity, calculated from the increase in ethanol concentration during the first $30 \mathrm{~min}$ of the experiments, was expressed as mmol ethanol produced (g of dry yeast biomass) $)^{-1} \mathrm{~h}^{-1}$. During the assay period, the increase in biomass concentration was negligible, and the increase in ethanol concentration was linear with time and proportional to the amount of biomass added.

\section{Transcriptome analysis}

Microarray analysis was performed with samples from independent triplicate steady-state chemostat cultures and duplicate retentostat cultures of $S$. cerevisiae strain CEN.PK113-7D sampled at 5 time points, comprising a total dataset of 13 microarrays. Sampling for transcriptome analysis was carried out by using liquid nitrogen for rapid quenching of mRNA turnover [72]. Prior to RNA extraction, samples were stored in a mixture of phenol/chloroform and TEA buffer at $-80{ }^{\circ} \mathrm{C}$. Total RNA 
extraction, isolation of mRNA, cDNA synthesis, cRNA synthesis, labelling and array hybridization was performed as described previously [73], with the following modifications. The quality of total RNA, cDNA, aRNA and fragmented aRNA was checked using an Agilent Bioanalyzer 2100 (Agilent Technologies, Santa Clara, CA). Hybridization of labelled fragmented aRNA to the microarrays and staining, washing and scanning of the microarrays was performed according to Affymetrix instructions (EukGE_WS2v5).

The 6383 yeast open reading frames were extracted from the 9335 transcript features on the YG-S98 microarrays. To allow comparison, all expression data were normalized to a target value of 240 using the average signal from all gene features. To eliminate variation in genes that are essentially not expressed, genes with expression values below 12 were set to 12 and the genes for which the average expression was below 20 for all 13 arrays were discarded. The coefficient of variation of the mean transcript data of replicate retentostats was approximately $10 \%$, similar to the reproducibility usually observed in replicate steady state chemostat cultures [74]. The expression of housekeeping genes ACT1, HHT2, SHR3, PDA1, TPI and TFC1 [75] remained stable for both strains at all tested growth rates (average coefficient of variation $11 \pm 4 \%$ see Additional file 13 ).

To perform a differential expression analysis based on gene expression profiles across the different growth rates, EDGE version 1.1.291 [76] was used with growth rate as covariate. Genes with expression profiles with a $\mathrm{p}$ value below 0.01 were considered to significantly correlate with growth rate, and were clustered with k-means clustering using consensus clustering (GenePattern 2.0, Broad Institute, [77]). For display of specific growth rate dependent expression profiles, expression values were normalized per gene by dividing single expression values by the average expression value at all different growth rates. Averages \pm standard deviation of these average-normalized values are shown in Figs. 5, 6, 7 and 8.

Gene expression clusters were analysed for overrepresentation of functional annotation categories from the Munich Information Centre for Protein Sequences (MIPS) database (http://mips.gsf.de/genre/proj/yeast), the Gene Ontology (GO) database (http://geneontology.org/) and transcription factor binding (TF) according to [78], based on the hypergeometric distribution analysis tool described by Knijnenburg et al. [79]. Additional functional categories that were searched for enrichment originate from [30, 35, 80, 81] and are listed in Additional file 14.

\section{Additional files}

Additional file 1. Set of differentially expressed genes during the transition from exponential growth to stationary phase in aerobic batches of $S$. cerevisiae with glucose as carbon source [34], and overlap with the set of differentially expressed genes during aerobic retentostat cultures in the present study.

Additional file 2. Number of genes identified in cluster 1 and 2 that overlap with genes identified in previous growth studies of which the expression correlated with specific growth rate. "Repressed" indicates a positive correlation, "induced" indicates a negative correlation, referring to the mode of regulation at low specific growth rates. Numbers outside the circle indicate the number of genes identified by the corresponding study, but not identified in the present study.

Additional file 3. Genes with significant growth-rate dependent gene expression in aerobic and anaerobic cultures between specific growth rates of $0.1 \mathrm{~h}^{-1}$ and below $0.001 \mathrm{~h}^{-1}$ (see Fig. 6).

Additional file 4. Residual glucose concentrations and specific glucoseconsumption rate of aerobic and anaerobic cultures during prolonged retentostat. The data is represented as the average \pm standard deviation of replicate independent retentostat samples. The residual glucose concentration data point at $0.1 \mathrm{~h}^{-1}$ originates from aerobic glucose-limited chemostat cultures described in [82], operated and sampled under the same conditions as reported in the "Methods" section of this work.

Additional file 5. Three files encompassing the MATLAB model to predict biomass accumulation, specific growth rate, and specific glucose consumption rates over the course of retentostat (see "Methods" section). Additional file 5 describes how to run or adapt the prediction model. The model itself is contained in two Matlab files (Additional files 6 and 7).

Additional file 6. See Additional file 5 caption.Additional file 7. See Additional file 5 caption.

Additional file 8. Five files encompassing the MATLAB model for nonlinear regression of biomass accumulation in retentostat (see "Methods" section). Additional file 8 describes how to run or adapt the regression model. Additional file 9 is an example Excel file that can be adapted to include (hypothetical) experimental data. In addition, MATLAB writes the output of the regression model to this Excel file. The model itself is contained in three Matlab files (Additional files 10,11 and 12) which operate together with Additional file 9.

Additional file 9. See Additional file 8 caption.

Additional file 10. See Additional file 8 caption.

Additional file 11. See Additional file 8 caption.

Additional file 12. See Additional file 8 caption.

Additional file 13. Averaged normalized gene expression of housekeeping genes for $S$. cerevisiae strain CEN.PK113-7D [75]. Dotted bars indicate $20 \%$ variation around normalized expression.

Additional file 14. List of gene-groups described in literature that were mined for enrichment among differentially expressed genes identified in clusters 1 and 2 in Fig. 6.

\section{Abbreviations}

$\mu$ : specific growth rate; $q_{p}$ : specific production rate; $q_{s}$ : specific substrate consumption rate; $Y_{X / s}^{\max }$ : maximum biomass yield on substrate; $m_{s}$ : specific rate of substrate consumption for maintenance; $m_{\text {ATP }}$ : specific rate of ATP consumption for maintenance; $C_{\text {s: }}$ growth-limiting substrate (glucose) concentration; $C_{S, M c}$ : glucose concentration in medium reservoir during chemostat phase; $C_{S, M R}$ : glucose concentration in medium reservoir during retentostat phase; $V_{S}$ : volume mixing vessel; $\Phi_{V}$ : flow rate. 


\begin{abstract}
Authors' contributions
TV carried out the chemostat and retentostat studies, whole genome transcriptome analysis and contributed to writing the manuscript. XDVH contributed to the retentostat studies and the modelling. EdH contributed to the operational design of the retentostat. AvM contributed to the operational design of the retentostat and the modelling. JTP participated in the design of the study and contributed to writing the manuscript. PDL participated in the design of the study, coordinated the project and contributed to writing the manuscript. All authors read and gave input to the final version of the manuscript. All authors read and approved the final manuscript.
\end{abstract}

\section{Acknowledgements}

We would like to thank Corinna Rebnegger, Rinke van Tatenhove-Pel and Pilar de la Torre Cortes for assistance in the experiments.

\section{Availability of data}

The transcriptome data have been made available at the GEO data repository under accession number GSE77842.

\section{Competing interests}

The authors declare that they have no competing interests.

\section{Funding}

This work was supported by the B-Basic research programme, which was financed by the Netherlands Organisation for Scientific Research (NWO), and by the European Union's Framework VII programme (RoBoYeast project).

Received: 24 February 2016 Accepted: 31 May 2016

Published online: 17 June 2016

\section{References}

1. Kavšček M, Stražar M, Curk T, Natter K, Petrovič U. Yeast as a cell factory: current state and perspectives. Microb Cell Fact. 2015;14:94. doi:10.1186/ s12934-015-0281-X.

2. Chemler JA, Yan YJ, Koffas MAG. Biosynthesis of isoprenoids, polyunsaturated fatty acids and flavonoids in Saccharomyces cerevisiae. Microb Cell Fact. 2006;5:20.

3. Vos T, Cortes PD, van Gulik WM, Pronk JT, Daran-Lapujade P. Growth-rate dependency of de novo resveratrol production in chemostat cultures of an engineered Saccharomyces cerevisiae strain. Microb Cell Fact. 2015;14:133. doi:10.1186/s12934-015-0321-6.

4. Liu ZH, Hou J, Martinez JL, Petranovic D, Nielsen J. Correlation of cell growth and heterologous protein production by Saccharomyces cerevisiae. Appl Microbiol Biotechnol. 2013;97:8955-62.

5. Van Dijken JP, Weusthuis RA, Pronk JT. Kinetics of growth and sugar consumption in yeasts. Antonie Van Leeuwenhoek. 1993;63:343-52.

6. van Hoek P, de Hulster E, van Dijken JP, Pronk JT. Fermentative capacity in high-cell-density fed-batch cultures of baker's yeast. Biotechnol Bioeng. 2000;68:517-23.

7. Hensing MCM, Rouwenhorst RJ, Heijnen JJ, Dijken JP, Pronk JT. Physiological and technological aspects of large-scale heterologous-protein production with yeasts. Antonie Van Leeuwenhoek. 1995;67:261-79.

8. Kocharin K, Nielsen J. Specific growth rate and substrate dependent polyhydroxybutyrate production in Saccharomyces cerevisiae. AMB Express. 2013:3:18.

9. Johansson N, Quehl P, Norbeck J, Larsson C. Identification of factors for improved ethylene production via the ethylene forming enzyme in chemostat cultures of Saccharomyces cerevisiae. Microb Cell Fact. 2013;1 2:89.

10. Tannler S, Decasper S, Sauer U. Maintenance metabolism and carbon fluxes in Bacillus species. Microb Cell Fact. 2008;7:19. doi:10.1186/1475-2859-7-19.

11. Pirt SJ. Maintenance energy of bacteria in growing cultures. Proc R Soc Lond B Biol Sci. 1965;163:224-8.

12. Boender LGM, De Hulster EAF, van Maris AJA, Daran-Lapujade P, Pronk JT. Quantitative physiology of Saccharomyces cerevisiae at near-zero specific growth rates. Appl Environ Microbiol. 2009;75:5607-14.

13. Boender LGM, Almering MJH, Dijk M, van Maris AJA, de Winde JH, Pronk JT, Daran-Lapujade P. Extreme calorie restriction and energy source starvation in Saccharomyces cerevisiae represent distinct physiological states. Biochim Biophys Acta. 2011;1813:2133-44.

14. Boender LGM, van Maris AJA, Hulster E, Almering MJH, Klei IJ, Veenhuis M, Winde JH, Pronk JT, Daran-Lapujade P. Cellular responses of Saccharomyces cerevisiae at near-zero growth rates: transcriptome analysis of anaerobic retentostat cultures. FEMS Yeast Res. 2011;11:603-20.

15. Bisschops MMM, Zwartjens P, Keuter SGF, Pronk JT, Daran-Lapujade P. To divide or not to divide: a key role of Rim 15 in calorie-restricted yeast cultures. Biochim Biophys Acta. 2014;1843:1020-30.

16. Binai NA, Bisschops MMM, van Breukelen B, Mohammed S, Loeff L, Pronk JT, Heck AJR, Daran-Lapujade P, Slijper M. Proteome adaptation of Saccharomyces cerevisiae to severe calorie restriction in retentostat cultures. J Proteome Res. 2014;13:3542-53.

17. van Verseveld HW, Arbige M, Chesbro WR. Continuous culture of bacteria with biomass retention. Trends Biotechnol. 1984;2:8-12.

18. Ercan O, Bisschops MMM, Overkamp W, Jørgensen TR, Ram AF, Smid EJ, Pronk JT, Kuipers OP, Daran-Lapujade P, Kleerebezem M. Physiological and transcriptional responses of different industrial microbes at near-zero specific growth rates. Appl Environ Microbiol. 2015;81:5662-70.

19. van Verseveld HW, de Hollander J, Frankena J, Braster M, Leeuwerik FJ, Stouthamer AH. Modeling of microbial substrate conversion, growth and product formation in a recycling fermentor. Antonie Van Leeuwenhoek. 1986;52:325-42.

20. Arbige M, Chesbro WR. Very slow growth of Bacillus polymyxa-stringent response and maintenance energy. Arch Microbiol. 1982;132:338-44.

21. Arbige M, Chesbro W. Rela and related loci are growth-rate determinants for Escherichia coli in a recycling fermenter. J Gen Microbiol. 1982;128:693-703.

22. Lu C, Brauer MJ, Botstein D. Slow growth induces heat-shock resistance in normal and respiratory-deficient yeast. Mol Biol Cell. 2009;20:891-903.

23. Verduyn C, Stouthamer AH, Scheffers WA, van Dijken JP. A theoretical evaluation of growth yields of yeasts. Antonie Van Leeuwenhoek. 1991:59:49-63.

24. van Bodegom P. Microbial maintenance: a critical review on its quantification. Microb Ecol. 2007;53:513-23.

25. Verduyn C, Postma E, Scheffers WA, van Dijken JP. Physiology of Saccharomyces cerevisiae in anaerobic glucose-limited chemostat cultures. J Gen Microbiol. 1990;136:395-403.

26. Jamieson DJ. Oxidative stress responses of the yeast Saccharomyces cerevisiae. Yeast. 1998;14:1511-27.

27. Chen QH, Ding QX, Keller RN. The stationary phase model of aging in yeast for the study of oxidative stress and age-related neurodegeneration. Biogerontology. 2005;6:1-13.

28. van Hoek P, van Dijken JP, Pronk JT. Regulation of fermentative capacity and levels of glycolytic enzymes in chemostat cultures of Saccharomyces cerevisiae. Enzyme Microb Tech. 2000;26:724-36.

29. Famili I, Forster J, Nielsen J, Palsson BO. Saccharomyces cerevisiae phenotypes can be predicted by using constraint-based analysis of a genome-scale reconstructed metabolic network. Proc Natl Acad Sci USA. 2003;100:13134-9.

30. Gasch AP, Spellman PT, Kao CM, Carmel-Harel O, Eisen MB, Storz G, Botstein D, Brown PO. Genomic expression programs in the response of yeast cells to environmental changes. Mol Biol Cell. 2000;11:4241-57.

31. Castrillo J, Zeef L, Hoyle D, Zhang N, Hayes A, Gardner D, Cornell M, Petty J, Hakes L, Wardleworth L, et al. Growth control of the eukaryote cell: a systems biology study in yeast. J Biol. 2007;6:4.

32. Regenberg B, Grotkjær T, Winther O, Fausbø\|l A, Akesson M, Bro C, Hansen LK, Brunak S, Nielsen J. Growth-rate regulated genes have profound impact on interpretation of transcriptome profiling in Saccharomyces cerevisiae. Genome Biol. 2006;7:R107.

33. Brauer MJ, Huttenhower C, Airoldi EM, Rosenstein R, Matese JC, Gresham D, Boer VM, Troyanskaya OG, Botstein D. Coordination of growth rate, cell cycle, stress response, and metabolic activity in yeast. Mol Biol Cell. 2008;19:352-67.

34. Bisschops MMM, Vos T, Moreno-Martinez R, de la Torre-Cortes P, Pronk JT, Daran-Lapujade P. Oxygen availability strongly affects chronological lifespan and thermotolerance in batch cultures of Saccharomyces cerevisiae. Microbial Cell. 2015;2:429-44.

35. Eastmond DL, Nelson HCM. Genome-wide analysis reveals new roles for the activation domains of the Saccharomyces cerevisiae heat shock 
transcription factor ( $\mathrm{Hsf1}$ ) during the transient heat shock response. J Biol Chem. 2006;281:32909-21.

36. Petitjean M, Teste MA, Francois JM, Parrou JL. Yeast tolerance to various stresses relies on the trehalose-6P synthase (Tps1) protein, not on trehalose. J Biol Chem. 2015;290:16177-90.

37. Crowe JH. Trehalose as a "chemical chaperone": fact and fantasy. Adv Exp Med Biol. 2007;594:143-58.

38. van den Brink J, Daran-Lapujade P, Pronk JT, de Winde JH. New insights into the Saccharomyces cerevisiae fermentation switch: Dynamic transcriptional response to anaerobicity and glucose-excess. BMC Genomics. 2008:9:100. doi:10.1186/1471-2164-9-100.

39. Daran-Lapujade P, Rossell S, van Gulik WM, Luttik MAH, de Groot MJL, Slijper M, Heck AJR, Daran JM, de Winde JH, Westerhoff HV, et al. The fluxes through glycolytic enzymes in Saccharomyces cerevisiae are predominantly regulated at posttranscriptional levels. Proc Natl Acad Sci USA. 2007;104:15753-8.

40. van Hoek P, van Dijken JP, Pronk JT. Effect of specific growth rate on fermentative capacity of baker's yeast. Appl Environ Microbiol. 1998;64:4226-33.

41. Solis-Escalante D, Kuijpers NGA, Barrajon-Simancas N, van den Broek M, Pronk JT, Daran JM, Daran-Lapujade P. A minimal set of glycolytic genes reveals strong redundancies in Saccharomyces cerevisiae central metabolism. Eukaryot Cell. 2015;14:804-16.

42. Schrickx JM, Raedts MJH, Stouthamer AH, van Verseveld HW. Organic-acid production by Aspergillus niger in recycling culture analyzed by capillary electrophoresis. Anal Biochem. 1995;231:175-81.

43. Schrickx JM, Stouthamer AH, van Verseveld HW. Growth-behavior and glucoamylase production by Aspergillus niger N402 and a glucoamylase overproducing transformant in recycling culture without a nitrogensource. Appl Microbiol Biotechnol. 1995;43:109-16.

44. Chesbro W, Evans T, Eifert R. Very slow growth of Escherichia coli. J Bacteriol. 1979;139:625-38.

45. Rogers PJ, Stewart PR. Energetic efficiency and maintenance energy characteristics of Saccharomyces cerevisiae (wild-type and petite) and Candida parapsilosis grown aerobically and microaerobically in continuous culture. J Appl Chem Biotechnol. 1976;26:335-6.

46. Sheldon JG, Williams SP, Fulton AM, Brindle KM. P-31 NMR magnetization transfer study of the control of ATP turnover in Saccharomyces cerevisiae. Proc Natl Acad Sci USA. 1996;93:6399-404.

47. van Rolleghem PA, de Jong-Gubbels P, van Gulik WM, Pronk JT, van Dijken JP, Heijnen S. Validation of a metabolic network for Saccharomyces cerevisiae using mixed substrate studies. Biotechnol Prog. 1996;12:434-48.

48. Piper P, Calderon CO, Hatzixanthis K, Mollapour M. Weak acid adaptation: the stress response that confers yeasts with resistance to organic acid food preservatives. Microbiology. 2001;147:2635-42.

49. Verduyn C, Postma E, Scheffers WA, van Dijken JP. Effect of benzoicacid on metabolic fluxes in yeasts-a continuous-culture study on the regulation of respiration and alcoholic fermentation. Yeast. 1992:8:501-17.

50. Abbott DA, Knijnenburg TA, de Poorter LM, Reinders MJ, Pronk JT, van Maris AJA. Generic and specific transcriptional responses to different weak organic acids in anaerobic chemostat cultures of Saccharomyces cerevisiae. FEMS Yeast Res. 2007:7:819-33.

51. Schmidt FR. Optimization and scale up of industrial fermentation processes. Appl Microbiol Biotechnol. 2005;68:425-35.

52. Gibson BR, Lawrence SJ, Leclaire JPR, Powell CD, Smart KA. Yeast responses to stresses associated with industrial brewery handling. FEMS Microbiol Rev. 2007;31:535-69.

53. De Virgilio C, Hottiger T, Dominguez J, Boller T, Wiemken A. The role of trehalose synthesis for the acquisition of thermotolerance in yeast, genetic evidence that trehalose is a thermoprotectant. FEBS J. 1994;219:179-86.

54. Jules M, Beltran G, Francois J, Parrou JL. New insights into trehalose metabolism by Saccharomyces cerevisiae: NTH2 encodes a functional cytosolic trehalase, and deletion of TPS1 reveals Ath $1 \mathrm{p}$-dependent trehalose mobilization. Appl Environ Microbiol. 2008;74:605-14.

55. Shi L, Sutter BM, Ye XY, Tu BP. Trehalose is a key determinant of the quiescent metabolic state that fuels cell cycle progression upon return to growth. Mol Biol Cell. 2010;21:1982-90.

56. Caspeta L, Chen Y, Ghiaci P, Feizi A, Buskov S, Hallstrom BM, Petranovic D, Nielsen J. Altered sterol composition renders yeast thermotolerant. Science. 2014;346:75-8.
57. Wilson K, McLeod BJ. The influence of conditions of growth on the endogenous metabolism of Saccharomyces cerevisiae: effect on protein, carbohydrate, sterol and fatty acid content and on viability. Antonie Van Leeuwenhoek. 1976;42:397-410.

58. Stouthamer AH. Theoretical study on amount of ATP required for synthesis of microbial cell material. Antonie Van Leeuwenhoek. 1973;39:545-65.

59. de Groot MJL, Daran-Lapujade P, van Breukelen B, Knijnenburg TA, De Hulster EAF, Reinders MJT, Pronk JT, Heck AJR, Slijper M. Quantitative proteomics and transcriptomics of anaerobic and aerobic yeast cultures reveals post-transcriptional regulation of key cellular processes. Microbiology. 2007;153:3864-78.

60. Christiano R, Nagaraj N, Frohlich F, Walther TC. Global proteome turnover analyses of the yeasts S. cerevisiae and S. pombe. Cell Reports. 2014;9:1959-65.

61. Jansen MLA, Diderich JA, Mashego M, Hassane A, de Winde JH, DaranLapujade P, Pronk JT. Prolonged selection in aerobic, glucose-limited chemostat cultures of Saccharomyces cerevisiae causes a partial loss of glycolytic capacity. Microbiology. 2005;151:1657-69.

62. Partow S, Siewers V, Bjorn S, Nielsen J, Maury J. Characterization of different promoters for designing a new expression vector in Saccharomyces cerevisiae. Yeast. 2010;27:955-64.

63. Fazio A, Jewett MC, Daran-Lapujade P, Mustacchi R, Usaite R, Pronk JT, Workman CT, Nielsen J. Transcription factor control of growth rate dependent genes in Saccharomyces cerevisiae: a three factor design. BMC Genom. 2008;9:341.

64. Monod J. The growth of bacterial cultures. Annu Rev Microbiol. 1949:3:371-94

65. Kresnowati MT, van Winden W, Almering MJH, Ten P, Ras C, Knijnenburg T, Daran-Lapujade P, Pronk JT, Heijnen JJ, Daran JM. When transcriptome meets metabolome: fast cellular responses of yeast to sudden relief of glucose limitation. Mol Sys Biol. 2006;2:49.

66. Entian KD, Kotter P. Yeast genetic strain and plasmid collections. Yeast Gene Anal Second Edit. 2007;36:629-66.

67. Nijkamp JF, van den Broek M, Datema E, de Kok S, Bosman L, Luttik MA, Daran-Lapujade P, Vongsangnak W, Nielsen J, Heijne WHM, et al. De novo sequencing, assembly and analysis of the genome of the laboratory strain Saccharomyces cerevisiae CEN.PK113-7D, a model for modern industrial biotechnology. Microb Cell Fact. 2012;11:36.

68. Daran-Lapujade P, Daran JM, Luttik MAH, Almering MJH, Pronk JT, Kotter P. An atypical PMR2 locus is responsible for hypersensitivity to sodium and lithium cations in the laboratory strain Saccharomyces cerevisiae CEN. PK113-7D. FEMS Yeast Res. 2009:9:789-92.

69. Pirt SJ. Maintenance energy-a general model for energy-limited and energy-sufficient growth. Arch Microbiol. 1982;133:300-2.

70. Mashego MR, van Gulik WM, Vinke JL, Heijnen JJ. Critical evaluation of sampling techniques for residual glucose determination in carbonlimited chemostat culture of Saccharomyces cerevisiae. Biotechnol Bioeng. 2003:83:395-9.

71. Parrou JL, Francois J. A simplified procedure for a rapid and reliable assay of both glycogen and trehalose in whole yeast cells. Anal Biochem. 1997:248:186-8.

72. Piper MD, Daran-Lapujade P, Bro C, Regenberg B, Knudsen S, Nielsen J, Pronk JT. Reproducibility of oligonucleotide microarray transcriptome analyses. An interlaboratory comparison using chemostat cultures of Saccharomyces cerevisiae. J Biol Chem. 2002;277:37001-8.

73. Mendes F, Sieuwerts S, de Hulster E, Almering MJH, Luttik MAH, Pronk JT, Smid EJ, Bron PA, Daran-Lapujade P. Transcriptome-based characterization of interactions between Saccharomyces cerevisiae and Lactobacillus delbrueckii subsp bulgaricus in lactose-grown chemostat cocultures. Appl Environ Microbiol. 2013;79:5949-61.

74. Daran-Lapujade P, Jansen ML, Daran J-M, van GulikW, de Winde JH, Pronk JT. Role of transcriptional regulation in controlling fluxes in central carbon metabolism of Saccharomyces cerevisiae. A chemostat culture study. J Biol Chem. 2004;279:9125-38.

75. Teste MA, Duquenne M, Francois JM, Parrou JL. Validation of reference genes for quantitative expression analysis by real-time RT-PCR in Saccharomyces cerevisiae. BMC Mol Biol. 2009;10:99.

76. Magoc T, Wood D, Salzberg SL. EDGE-pro: Estimated Degree of Gene Expression in prokaryotic genomes. Evol Bioinform. 2013;9:127-36.

77. Reich M, Liefeld T, Gould J, Lerner J, Tamayo P, Mesirov JP. GenePattern 2.0. Nat Genet. 2006;38:500-1. 
78. Harbison CT, Gordon DB, Lee TI, Rinaldi NJ, Macisaac KD, Danford TW, Hannett NM, Tagne JB, Reynolds DB, Yoo J, et al. Transcriptional regulatory code of a eukaryotic genome. Nature. 2004;431:99-104.

79. Knijnenburg TA, de Winde JH, Daran J-M, Daran-Lapujade P, Pronk JT, Reinders MJ, Wessels LF. Exploiting combinatorial cultivation conditions to infer transcriptional regulation. BMC Genom. 2007;8:25.

80. Verghese J, Abrams J, Wang YY, Morano KA. Biology of the heat shock response and protein chaperones: budding yeast (Saccharomyces cerevisiae) as a model system. Microbiol Mol Biol Rev. 2012;76:115-58.
81. Gibney PA, Lu C, Caudy AA, Hess DC, Botstein D. Yeast metabolic and signaling genes are required for heat-shock survival and have little overlap with the heat-induced genes. Proc Natl Acad Sci USA. 2013;110:E4393-402.

82. Kozak BU, van Rossum HM, Benjamin KR, Wu L, Daran J-M, Pronk JT, van Maris AJA. Replacement of the Saccharomyces cerevisiae acetyl-CoA synthetases by alternative pathways for cytosolic acetyl-CoA synthesis. Metab Eng. 2014;21:46-59.

\section{Submit your next manuscript to BioMed Central and we will help you at every step:}

- We accept pre-submission inquiries

- Our selector tool helps you to find the most relevant journal

- We provide round the clock customer support

- Convenient online submission

- Thorough peer review

- Inclusion in PubMed and all major indexing services

- Maximum visibility for your research

Submit your manuscript at

www.biomedcentral com/submit 